\title{
Deep Brain Stimulation for the Treatment of Resistant Depression: Systematic Review of the Literature
}

\section{Estimulação cerebral profunda para o tratamento de depressão resistente: revisão sistemática da literatura}

\author{
Ícaro Elias Gualberto Santos Julião ${ }^{1}$ Diego Ferreira Santana ${ }^{1}$ Ledismar José da Silva ${ }^{10}$ \\ ${ }^{1}$ Department of Medicine, Pontifícia Universidade Católica de Goiás, \\ Goiânia, GO, Brazil \\ Arq Bras Neurocir 2019;38:183-198.

\begin{abstract}
Address for correspondence Ledismar José da Silva, MSc, Pontifícia Universidade Católica de Goiás (PUC-Goiás), Avenida Universitária, 1440, Setor Universitário, 74605-010, Goiânia, GO, Brazil (e-mail: ledismarsilva@gmail.com).
\end{abstract}

\begin{abstract}
Depression is the leading cause of disability worldwide, and it is related to high suicide rates. Furthermore, a great number of patients do not respond to any of the available treatments. Deep brain stimulation (DBS), a versatile technology with expanding

Keywords

- deep brain stimulation

- treatment-resistant depression

- refractory depression

Resumo

Palavras-chave

- estimulação cerebral profunda

- depressão resistente ao tratamento

- depressão refratária indications, is considered a potential treatment for resistant depression. However, in over 10 years of clinical research, its efficacy has not been completely proven. Although new trials using DBS for treatment-resistant depression keep emerging, two of the three Level I evidence-based studies recently conducted have not provided conclusive data. Methodological limitations and major biases have compromised the obtention of clearer results. In this systematic review of the literature, we intend to critically assess the clinical trials performed in this field.

A depressão é a maior causa de incapacitação em nível mundial, e ela está relacionada com altos índices de suicídio. Ademais, um grande número de pacientes não responde a nenhum dos tratamentos disponíveis. A estimulação cerebral profunda (ECP), uma técnica versátil com indicações em expansão, é considerada um tratamento potencial para depressão refratária. Contudo, em mais de 10 anos de pesquisas clínicas, sua eficácia ainda não foi completamente comprovada. Embora novos estudos utilizando ECP para tratamento da depressão refratária venham sendo realizados, dois dos três ensaios recentemente conduzidos baseados em evidência com Nível 1 não forneceram dados conclusivos. Limitações metodológicas e vieses importantes comprometeram a obtenção de resultados mais claros. Nesta revisão sistemática da literatura, pretendemos avaliar criticamente as pesquisas clínicas executadas nesta área.
\end{abstract}

\section{Introduction}

Depression is a severe psychiatric disorder, presently recognized as the most frequent mental illness and the leading

(1DLedismar José da Silva's ORCID is https://orcid.org/0000-0002-35512650.

received

November 27, 2018

accepted

April 17, 2019 10.1055/s-0039-1692158. ISSN 0103-5355. cause of disability worldwide. ${ }^{1}$ It currently affects 260 million people (3.6\% of the global population) and is 1.5 to 2 times more common in women. ${ }^{2}$ However, multimodal treatments have often failed in up to $30 \%$ of the patients, ${ }^{3}$ a group considered to have treatment-resistant depression (TRD), ${ }^{4}$ which exhibits a 2-fold suicide risk. ${ }^{5}$ Globally, depression is the $2^{\text {nd }}$ cause of death among 15 to 29 -year-olds, ${ }^{6}$

Copyright $\odot 2019$ by Thieme Revinter Publicações Ltda, Rio de Janeiro, Brazil

License terms 
with alarming completed suicide rates of approximately 800 thousand per year, that is, 2,191 daily deaths.

Electroconvulsive therapy (ECT) is the most effective somatic treatment for depression, since it promotes remission rates $>40 \% .^{7,8}$ In spite of being effective, $\sim 52 \%$ of the patients resistant to antidepressants (ADs) do not respond to ECT either. ${ }^{9}$

Deep brain stimulation (DBS) consists in modulating deep brain structures through electrodes implanted using the stereotactic technique, ${ }^{10}$ and it has also been used for the treatment of depression. It is reversible, adjustable, and can be applied in combination with ADs. Moreover, the simultaneous use of DBS and ADs allows titration of both methods. Nonetheless, efficacy, optimal targets, and stimulation parameters (frequency, amplitude, pulse width, duration) for TRD remain unclear. ${ }^{11,12}$ The success of DBS for the treatment of Parkinson disease (PD), dystonia, obsessive compulsive disorder (OCD), and, more recently, epilepsy, points to the versatility of this surgical procedure in clinical settings, instigating laser-focused research for TRD. Therefore, the present systematic review of the literature aimed to critically assess clinical evidence of DBS for TRD.

\section{Historical Remarks}

Depression has long been known, ${ }^{13}$ and so has neuromodulation through electricity and invasive brain procedures, inasmuch as Hippocrates (469-399 BCE) described trepanation for the management of intracranial hypertension. ${ }^{14,15}$ Ancient Greek doctors modulated pain with electric eels. ${ }^{16}$ The Greek word for these fish is narka, meaning "relief from pain", the root of the word narkoun, meaning "to benumb", which, in turn, is the root of the term "narcotics". 17

Scribonius Largus (1-50 CE) described the use of the shocks of Torpedo nobiliana, a species of electric ray, for headaches and gout derived chronic pain. This therapy drew the attention of Galen (130-210 CE), and this started a "torpedonism" trend described in several medical documents, including the Canon of Medicine, written by Avicenna, where this treatment was proposed for melancholy. ${ }^{18-20}$ This mental state is related to the melancholic depressive subtype and anhedonia, a core symptom of major depressive disorder (MDD) ${ }^{21}$ associated with the reward circuitry. ${ }^{22}$ Accessing the brain had further indications in other parts of the world, such as in Peru, where witch doctors (ca. 1000-1250 CE) employed this procedure to release bad spirits and treat mental illnesses. ${ }^{14}$

More recently, ablative surgery preceded in 30 years the advent of the first psychiatric drugs, that is, antipsychotics. ${ }^{23}$ The stereotaxy apparatus brought minimally invasive procedures, allowing the access to subcortical, deep brain structures. ${ }^{24}$ The term psychiatric neurosurgery, ${ }^{25}$ fitting the idea of recognized dysfunctional circuits in the brain, emerged in the $20^{\text {th }}$ century. ${ }^{23}$ A pioneer trial targeting the subgenual anterior cingulate cortex (SACC) inaugurated the modern era of neurosurgery for TRD, ${ }^{26}$ labelled later by the main investigator in the field as "keeping an eye on a moving target". 27

\section{Putative Neurocircuitry and Pathophysiology of Depression}

Although the taxonomy of psychiatric disorders is still incipient, knowledge of the underlying biology of depression has expanded from the concept of a disease purely correlated to limbic structures ${ }^{28}$ to a mental disorder involving several neural networks. ${ }^{29}$ Cortical structures thought to be implicated in depression involve several Brodmann areas (BAs), such as the dorsomedial prefrontal cortex (DMPFC), the medial prefrontal cortex (MPFC), the dorsolateral prefrontal cortex (DLPFC), the dorsal anterior cingulate cortex (dACC), the anterior cingulate cortex (ACC), the ventromedial prefrontal cortex ( $\mathrm{VmPFC}$ ), and the globus pallidus pars interna (GPI). ${ }^{14,29,30}$ The DLPFC and the orbitofrontal cortex (OFC) ${ }^{31}$ are connected to subcortical structures, such as the hippocampus, the amygdala nuclei, and the nucleus accumbens (NAcc). ${ }^{32}$ The cingulate gyrus and the hippocampus connect the vmPFC to the DLPFC. Furthermore, the hippocampus is intimately linked, both anatomically and physiologically, to the hypothalamus through the fornix, an axonal bundle that inhibits the hypothalamic pituitary adrenal (HPA) axis. ${ }^{32}$

Neoneurogenesis in the hippocampus is stimulated by monoaminergic agonists (e.g. selective serotonin reuptake inhibitors) and brain neurotrophins (e.g. brain derived neutrophic factor [BDNF]), and negatively modulated by stress, corticosteroids, and glutamatergic agents. ${ }^{32}$ In patients presenting with depression, the prefrontal cortex (PFC) would fail to inhibit overactive limbic structures implying cognitive, behavioral, mood, neuroendocrinal, pain modulation, and neurotransmitter activities due to its connection to the hypothalamus and the midbrain, notably the periaqueductal gray area. ${ }^{33}$ Rumination, suicidality, and complex symptoms suggest dysfunction of neural networks, rather than targets, ${ }^{34}$ outreaching the domain of anatomical/structural (overactive OFC/vmPFC, ACC, hippocampus, and amygdala, and hypoactive DLPFC), molecular (increased cortisol, corticotropin-releasing hormone, proinflammatory cytokines, decreased BDNF, serotonin, and noradrenalin), or cellular alterations (neurons, neural ensemble, ${ }^{35}$ and glia). ${ }^{32,36}$ It is believed that a major factor, yet to be unveiled, would trigger a cyclic "short-circuiting" in susceptible individuals, ${ }^{32}$ relying on a substrate of genetic predisposition, ${ }^{32}$ personal history, and affective temperament. ${ }^{29}$ That would ultimately disrupt adequate neurotransmission, neuroendocrine response, autonomic response, and cognitive function.

To date, putative DBS targets for the treatment of TRD include:

1. Subcallosal cingulate gyrus ( $\mathrm{SCg}$ ): also called subgenual cingulate gyrus, subcallosal cingulum, or SCg25 in the context of DBS TRD trials, it is the portion of the ACC lying ventrally to the corpus callosum, below its genu. ${ }^{37}$ It corresponds primarily to BA 25 , as well as to the caudal portions of BA 32 and of the inferior BA $24 .{ }^{26,29,38}$ The converging region in the $\mathrm{SCg}$ implicated in the response to fluoxetine $^{39}$ was chosen as the first DBS experimental target. $^{35}$ 
2. Ventral capsule/ventral striatum (VC/VS)/ventral anterior limb of the internal capsule (vALIC)/NAcc: The VC/VS comprehends a target region considered related to the pathophysiology of OCD and depression. ${ }^{40}$ The vALIC contains the prefrontal corticopontine tract and the anterior thalamic radiation (ATR), interconnecting MPFC and cingulate gyrus with the anterior and dorsomedial thalamic nuclei ${ }^{41}$ both extensively connected with the cortical and subcortical limbic areas, ${ }^{29,41}$ a functional link between the frontal lobe and the thalamus. The NAcc is a component of the VS linked to the ventral tegmental area (VTA), the amygdala, the hippocampus, the OFC, the mPFC, the motor territories of the caudate nucleus, and the GPI. ${ }^{42}$ Moreover, the NAcc is indirectly connected to the SCg and to the MPFC, and acts as "hub", amplifying or decreasing the signals from emotion centers. ${ }^{43}$

3. Bed nucleus of the stria terminalis (BNST): located in the adjacencies of the $\mathrm{VC} / \mathrm{VS}$ and NAcc regions, partially overlapping the VC/VS but distinct from it, it is an output pathway of the amygdala, and it regulates anxiety and threat vigilance, ${ }^{44}$ with projections to the medial forebrain bundle (MFB) and to the NAcc. ${ }^{45}$ The rationale for borrowing this target from OCD is that strong antidepressant effects appeared, particularly if the contacts were situated in or near the BNST. ${ }^{45}$

4. MFB: a white matter tract that mediates connectivity to the VTA and the NAcc, the hypothalamus (medial and lateral), the preoptic regions (lateral and medial), and the BNST. Its anatomical and functional connectivities have been described in diffusion tensor imaging studies. ${ }^{46,47}$ The MFB hypothetically mediates positive emotions ${ }^{48}$ particularly through the superolateral branch of the medial forebrain bundle (slMFB), and opposes the negative emotion modulation of ATR. The VTA is a key node of the reward circuit, mostly through dopamine. Rat models for optogenetics evidenced dopamine cell firing from the VTA. ${ }^{49}$

5. Inferior thalamic peduncle (ITP): a fiber bundle connecting the nonspecific thalamic nuclei/dorsomedial thala- mus (midline, intralaminar, and paralaminar) to the OFC. Subcaudate tractotomy includes the ITP and is classically described to treat TRD. Hypothetically, metabolic abnormalities in the frontal cortical regions are associated with depression, which could be modulated by employing DBS of the ITP. ${ }^{50}$

6. Lateral habenula ( $\mathrm{LHb})$ : is a brain structure projecting to several monoaminergic brainstem nuclei, involved in the metabolism of dopamine (substantia nigra pars compacta and VTA), serotonin (dorsal and medial raphe), ${ }^{51}$ and noradrenalin (locus coeruleus). ${ }^{52-54}$ Augmented activation in the nucleus of the LHb has been reported in depressed patients, ${ }^{55}$ and shown to downregulate neurotransmitters and stimulation of the HPA axis. ${ }^{56}$

\section{Methods}

A systematic review of the literature was carried out aiming to identify the efficacy of DBS for the treatment of TRD by two independent investigators, following the protocols of the Preferred Reporting Items for Systematic Review and MetaAnalysis (PRISMA). The databases searched for this review were: Australian New Zealand Clinical Trials Registry (ANZCTR), ClinicalTrials.gov, Cochrane Central Register of Controlled Trials (CENTRAL), Deutschen Register Klinischer Studien (DRKS), Instituto Brasileiro de Informação em Ciência e Tecnologia (IBICT), Latin American and Caribbean Health Sciences Literature (LILACS), Medical Literature, Analysis, and Retrieval System Online (MEDLINE), Netherlands Trial Registry (NTR), Portal de Periódicos da Coordenação de Aperfeiçoamento de Pessoal de Nível Superior do Ministério da Educação (CAPES/MEC), The Digital Library of Theses and Dissertations of the University of São Paulo (Digital Library USP), and World Health Organization International Clinical Trials Registry Platform (WHO ICTRP).

The following descriptors were used alone and with Boolean operators: depression, treatment-resistant depression, treatment-refractory depression, deep brain stimulation,

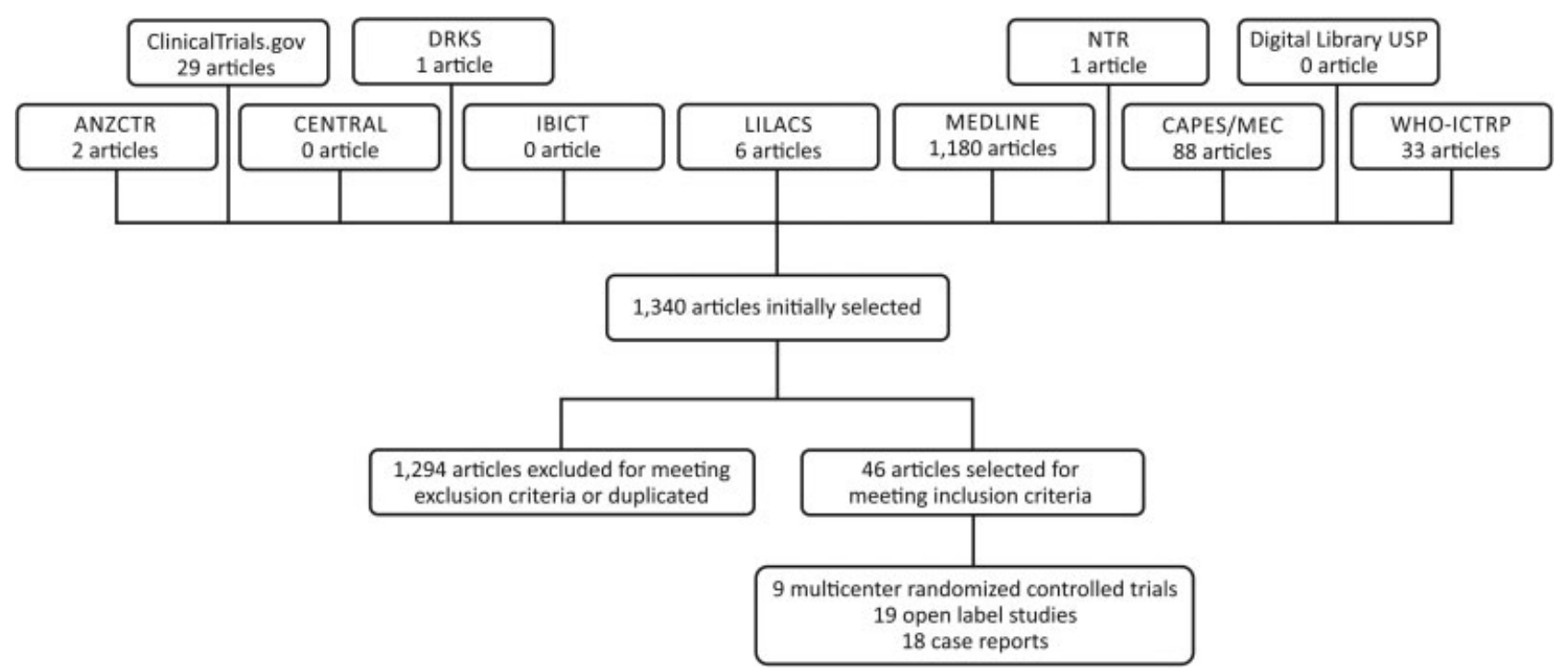

Fig. 1 Data collected during this systematic literature review. 


\begin{tabular}{|c|c|c|c|c|c|c|c|c|c|c|c|c|c|c|c|c|c|c|}
\hline \multicolumn{2}{|c|}{ 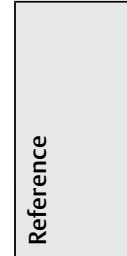 } & 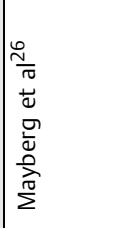 & 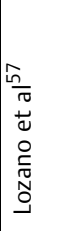 & 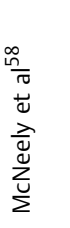 & 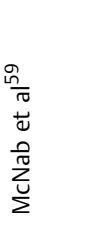 & 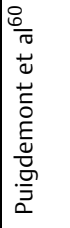 & 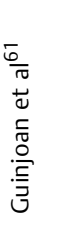 & 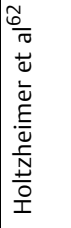 & 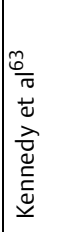 & 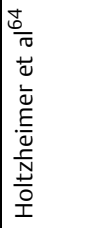 & 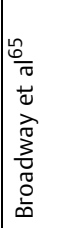 & 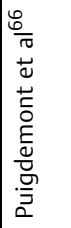 & 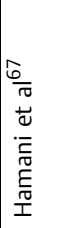 & 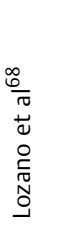 & 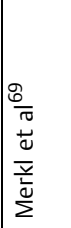 & 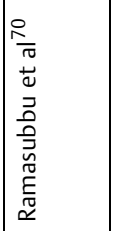 & 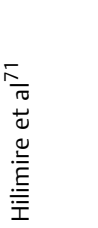 & 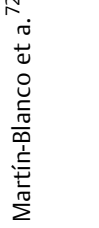 \\
\hline \multicolumn{2}{|c|}{ 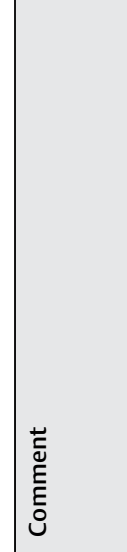 } & 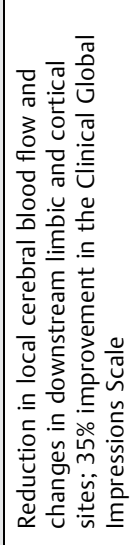 & 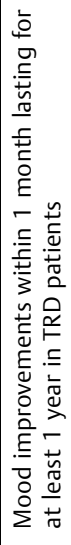 & 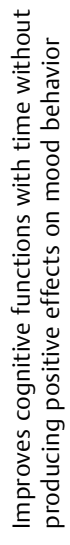 & 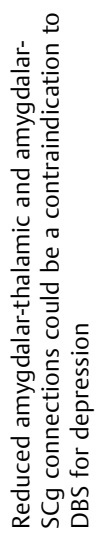 & 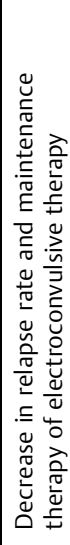 & 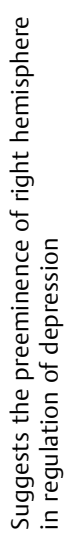 & 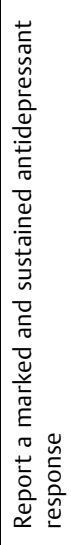 & 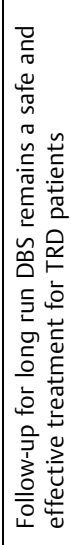 & 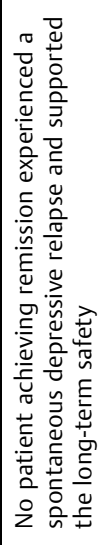 & 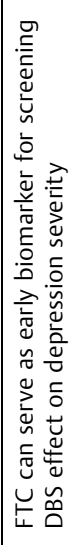 & 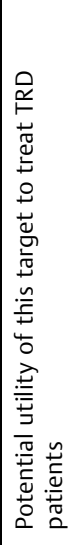 & 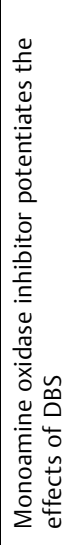 & 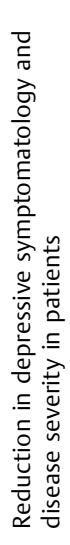 & 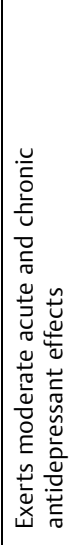 & 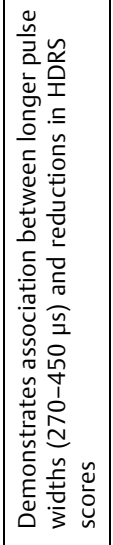 & 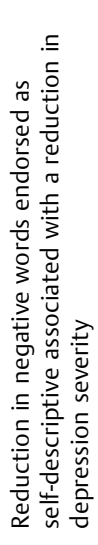 & 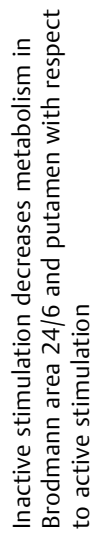 \\
\hline \multirow{2}{*}{ 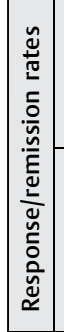 } & 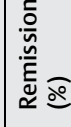 & in & $m$ & $\underline{z}$ & $\Sigma$ & 0 & $\frac{\mathbb{z}}{z}$ & $\Sigma$ & 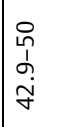 & $\stackrel{\infty}{\infty}$ & $\S$ & in & $\Sigma$ & $\frac{\pi}{z}$ & $\stackrel{m}{m}$ & in & $\underline{z}$ & 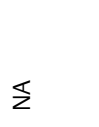 \\
\hline & 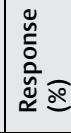 & $\stackrel{8}{8}$ & 㔯 & $\bullet$ & $\underline{z}$ & 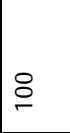 & $\Sigma$ & $\frac{s}{z}$ & f & $\sigma$ & $\ddot{6}$ & రิ & $\frac{s}{z}$ & $\stackrel{\curvearrowright}{N}$ & $\stackrel{m}{m}$ & in & $\sigma$ & $\underline{z}$ \\
\hline \multirow{4}{*}{ 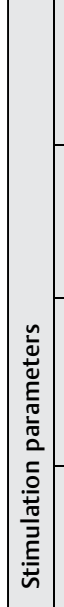 } & 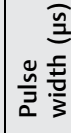 & 8 & 8 & 8 & $\bar{N}$ & 8 & ৪ & $\bar{\sigma}$ & 8 & $\bar{\sigma}$ & প & ৪ & 8 & \begin{tabular}{|l}
$\tilde{\infty}$ \\
$\vdots$ \\
1 \\
$\vdots$ \\
$\vdots$
\end{tabular} & ৪ & \begin{tabular}{|l}
0 \\
bq \\
0 \\
0 \\
0
\end{tabular} & $\bar{\sigma}$ & 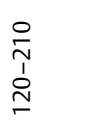 \\
\hline & 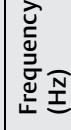 & m. & $\stackrel{p}{r}$ & $\stackrel{\stackrel{m}{r}}{ }$ & $\stackrel{\text { 号 }}{\stackrel{1}{n}}$ & $\stackrel{\stackrel{m}{m}}{\sim}$ & 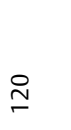 & $\stackrel{p}{\underline{m}}$ & m & $\stackrel{m}{m}$ & m & $\stackrel{\stackrel{m}{m}}{\sim}$ & $\stackrel{\stackrel{m}{m}}{ }$ & 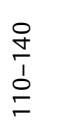 & m & $\begin{array}{l}\stackrel{\infty}{\infty} \\
\stackrel{1}{\sim} \\
\sim\end{array}$ & $\stackrel{m}{\check{m}}$ & $\stackrel{\text { m }}{\stackrel{m}{n}}$ \\
\hline & 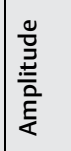 & $\begin{array}{l}\overrightarrow{3} \\
\stackrel{0}{+}\end{array}$ & \begin{tabular}{|l}
3 \\
0 \\
0 \\
1 \\
1 \\
مُ \\
$m$
\end{tabular} & $\begin{array}{l}\overrightarrow{3} \\
\dot{+} \\
\dot{j} \\
\dot{m} \\
\dot{m}\end{array}$ & $\begin{array}{l}\overrightarrow{3} \\
\text { iे }\end{array}$ & $\begin{array}{l}\vec{b} \\
\dot{m} \\
\dot{m}\end{array}$ & $\begin{array}{l}> \\
\stackrel{n}{+} \\
\text { ra }\end{array}$ & \begin{tabular}{|l}
$\hat{E}$ \\
$\hat{\varepsilon}$ \\
0 \\
$\dot{b}$
\end{tabular} & $\begin{array}{l}0 \\
0 \\
\hat{\rho} \\
\Lambda \\
n \\
m \\
m\end{array}$ & 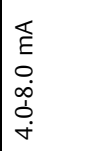 & \begin{tabular}{|l}
3 \\
0 \\
0 \\
0 \\
1 \\
0 \\
0
\end{tabular} & $\begin{array}{l}3 \\
0 \\
m\end{array}$ & in & $\begin{array}{l}> \\
0 \\
\dot{p} \\
\dot{1} \\
\hat{\omega} \\
m \\
m\end{array}$ & $\begin{array}{l}3 \\
0 \\
0 \\
0 \\
1 \\
\\
\sim\end{array}$ & \begin{tabular}{|l}
3 \\
$n$ \\
0 \\
0 \\
1 \\
0
\end{tabular} & 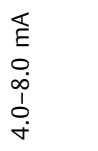 & 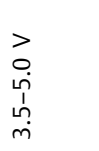 \\
\hline & 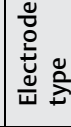 & $\begin{array}{l}\hat{\tilde{\Sigma}} \\
\bar{\infty}\end{array}$ & 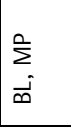 & $\frac{a}{\Sigma}$ & $\begin{array}{l}\text { ळे } \\
\dot{\infty}\end{array}$ & $\mid \begin{array}{l}\hat{\infty} \\
\vec{\infty}\end{array}$ & $\begin{array}{l}\sum_{\Sigma}^{2} \\
S^{\prime}\end{array}$ & $\mid \begin{array}{l}\tilde{\Sigma} \\
\vec{\omega} \\
\bar{\omega}\end{array}$ & $\mid \begin{array}{l}\tilde{\Sigma} \\
\tilde{\omega} \\
\tilde{\omega}\end{array}$ & $\mid \begin{array}{l}\tilde{y} \\
\bar{\omega} \\
\bar{\infty}\end{array}$ & $\mid \begin{array}{l}\tilde{y} \\
\bar{\omega} \\
\bar{\infty}\end{array}$ & $\begin{array}{l}\hat{\infty} \\
\vec{\infty}\end{array}$ & $\mid \begin{array}{l}\tilde{y} \\
\tilde{\infty} \\
\tilde{\infty}\end{array}$ & $\mid \begin{array}{l}\hat{y} \\
\dot{\infty}\end{array}$ & $\mid \begin{array}{l}\tilde{\Sigma} \\
\dot{\omega} \\
\tilde{\infty}\end{array}$ & $\mid \begin{array}{l}\tilde{\Sigma} \\
\tilde{\omega} \\
\tilde{\omega}\end{array}$ & $\begin{array}{l}\sum_{\Sigma}^{0} \\
\vec{\omega}\end{array}$ & $\mid \begin{array}{l}\tilde{n} \\
\dot{\omega} \\
\dot{\omega}\end{array}$ \\
\hline \multicolumn{2}{|c|}{ 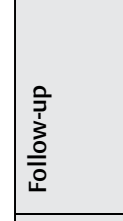 } & 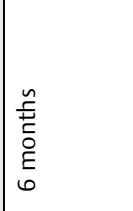 & 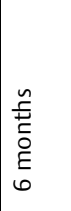 & 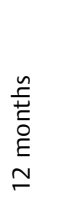 & 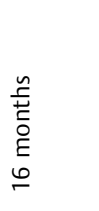 & 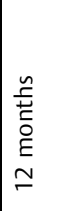 & 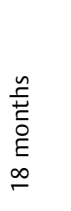 & 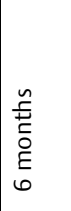 & 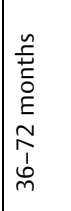 & 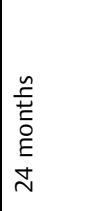 & 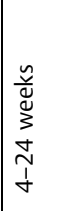 & 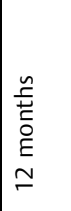 & \begin{tabular}{|l}
$\tilde{n}$ \\
$\underline{\underline{E}}$ \\
$\underline{\underline{0}}$ \\
$\underline{E}$ \\
6
\end{tabular} & 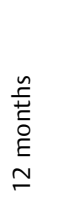 & 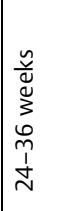 & 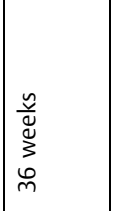 & 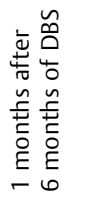 & 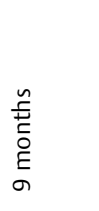 \\
\hline \multicolumn{2}{|c|}{$=$} & 0 & i & 0 & - & - & - & - & $\stackrel{\sim}{ }$ & 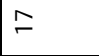 & $\simeq$ & $\infty$ & - & $\bar{N}$ & 0 & ナ & $\wedge$ & $\wedge$ \\
\hline \multicolumn{2}{|c|}{ 点 } & $\tilde{0}$ & 0 & 0 & $\stackrel{\sim}{\simeq}$ & Ч & ¿ & ک & 0 & 0 & o & 0 & $\stackrel{\sim}{\simeq}$ & 0 & 0 & $\overrightarrow{0}$ & 0 & ปै \\
\hline \multicolumn{2}{|c|}{ 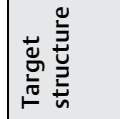 } & U్ & $\vec{\sim}$ & క్ & $\underset{n}{ }$ & u & " & 岕 & 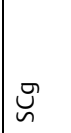 & $\vec{\sim}$ & 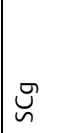 & $\breve{\sim}$ & un & $\breve{n}^{\prime}$ & $\breve{\sim}$ & ü & " & un \\
\hline
\end{tabular}




\begin{tabular}{|c|c|c|c|c|c|c|c|c|c|c|c|c|c|c|c|c|c|c|}
\hline$\stackrel{\breve{g}}{\mathrm{U}}$ & & 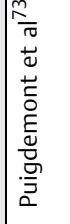 & 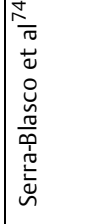 & 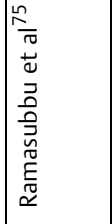 & 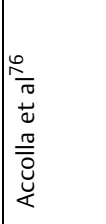 & 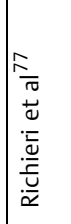 & 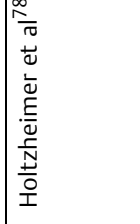 & 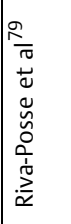 & 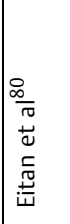 & 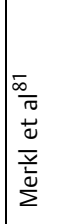 & 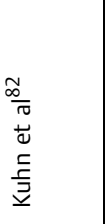 & 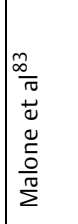 & 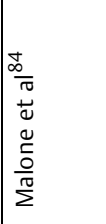 & 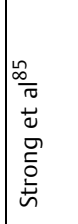 & 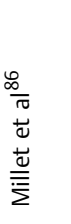 & 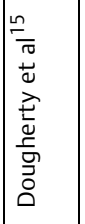 & 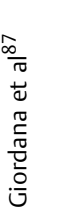 & 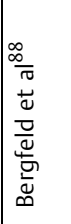 \\
\hline 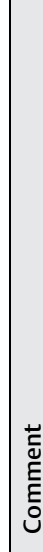 & & 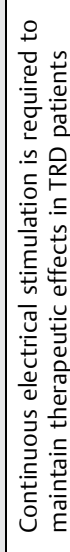 & 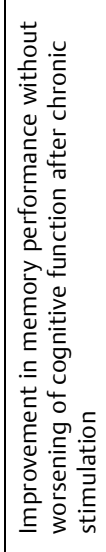 & 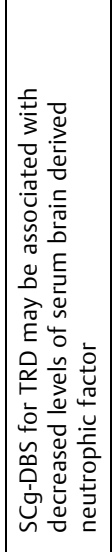 & 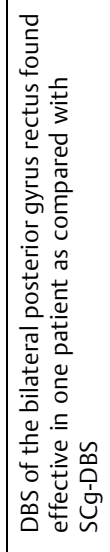 & 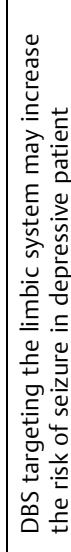 & 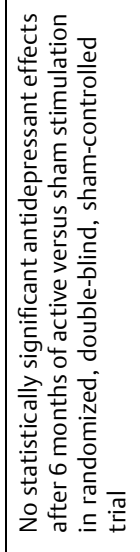 & 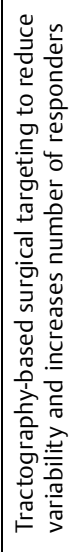 & 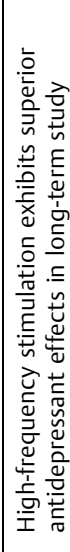 & 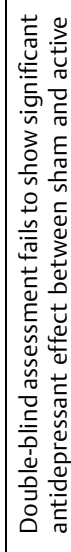 & 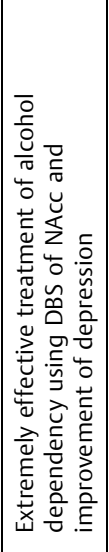 & 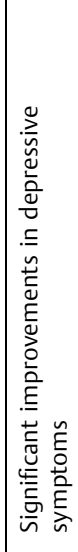 & 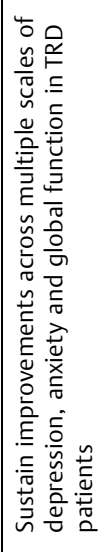 & 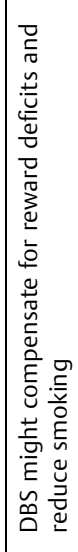 & 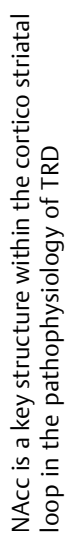 & 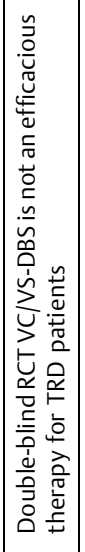 & 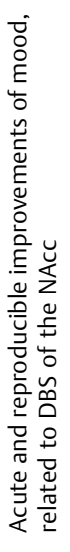 & 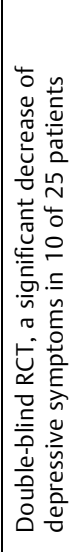 \\
\hline 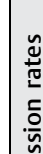 & 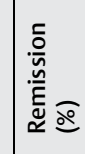 & 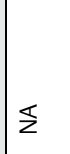 & $\stackrel{\nwarrow}{z}$ & $\S$ & $\stackrel{\sim}{\sim}$ & $\S$ & 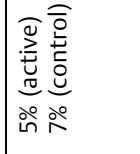 & 吉 & 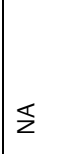 & $m$ & 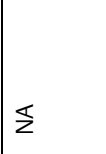 & ㅇ & $\stackrel{\stackrel{\leftrightarrow}{m}}{m}$ & 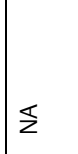 & $\underline{z}$ & i & $\Sigma$ & ì \\
\hline 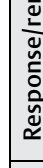 & 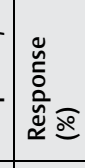 & $\frac{s}{z}$ & $\Sigma$ & in & $\stackrel{2}{1}$ & z & 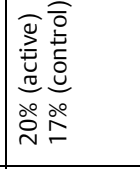 & $\dot{\infty}$ & $\ddot{\sim}$ & $\stackrel{m}{m}$ & $\underline{z}$ & ผ & i & 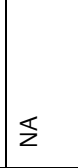 & $\underline{z}$ & 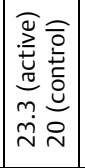 & $\Sigma$ & 宇 \\
\hline & 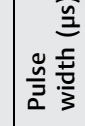 & 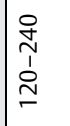 & 8 & 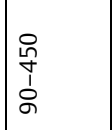 & 8 & 8 & $\bar{\sigma}$ & $\bar{\sigma}$ & б & ৪ & প & $\stackrel{\vec{m}}{F}$ & $\frac{\pi}{z}$ & $\stackrel{\mathbb{N}}{ }$ & 8 & $\begin{array}{l}\tilde{\hat{T}} \\
\grave{\alpha}\end{array}$ & ৪ & ৪ \\
\hline & 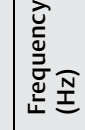 & $\begin{array}{l}\stackrel{n}{m} \\
\frac{m}{1} \\
\grave{m} \\
m\end{array}$ & $\stackrel{\stackrel{m}{m}}{\stackrel{2}{n}}$ & 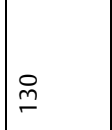 & $\stackrel{m}{m}$ & $\stackrel{m}{m}$ & m & $\stackrel{p}{r}$ & $\begin{array}{l}0 \\
\dot{m} \\
\vdots \\
\vdots \\
i \\
i\end{array}$ & $\stackrel{m}{m}$ & $\stackrel{p}{m}$ & $\stackrel{\nwarrow}{\simeq}$ & $\begin{array}{l}0 \\
0 \\
1 \\
o \\
0 \\
1\end{array}$ & $\stackrel{m}{m}$ & $\stackrel{m}{r}$ & $\Sigma$ & mి & $\begin{array}{l}0 \\
\infty \\
1 \\
0 \\
\end{array}$ \\
\hline 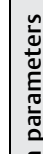 & 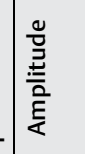 & $\begin{array}{l}> \\
0 \\
0 \\
\omega \\
\omega \\
\omega \\
m \\
m\end{array}$ & $\begin{array}{l}\vec{b} \\
\dot{m}\end{array}$ & $\begin{array}{l}3 \\
0 \\
i \\
\vdots \\
i \\
i\end{array}$ & $\begin{array}{l}3 \\
\text { Oे } \\
\text { in }\end{array}$ & $\underset{\sim}{\stackrel{\vec{f}}{+}}$ & $\begin{array}{l}\overleftarrow{\sigma} \\
\underline{E} \\
0 \\
o \\
0 \\
1 \\
\dot{\gamma} \\
\dot{\gamma}\end{array}$ & $\begin{array}{l}\sigma \\
\varepsilon \\
0 \\
\infty \\
0 \\
1 \\
0 \\
0\end{array}$ & 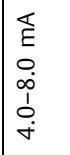 & $\begin{array}{l}> \\
0 \\
0 \\
0 \\
1 \\
\\
\\
\end{array}$ & 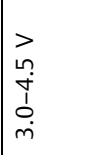 & $\mid \begin{array}{l}\lambda \\
\hat{\omega}\end{array}$ & $\begin{array}{l}3 \\
0 \\
0 \\
0 \\
1 \\
n \\
N\end{array}$ & $\begin{array}{l}3 \\
0 \\
\dot{b}\end{array}$ & $\begin{array}{l}> \\
0 \\
0 \\
0 \\
1 \\
\dot{+} \\
\dot{+}\end{array}$ & $\begin{array}{l}3 \\
0 \\
\infty\end{array}$ & $\begin{array}{l}> \\
0 \\
\dot{0} \\
\vdots \\
\dot{+} \\
\dot{+}\end{array}$ & $\begin{array}{l}3 \\
0 \\
b \\
1 \\
\omega \\
\sim \\
\sim\end{array}$ \\
\hline 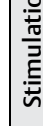 & 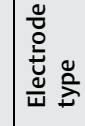 & $\mid \begin{array}{l}\tilde{\Sigma} \\
\dot{\omega} \\
\bar{\omega}\end{array}$ & 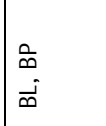 & $\begin{array}{l}\tilde{\Sigma} \\
\dot{\infty} \\
\bar{\infty}\end{array}$ & $\mid \begin{array}{l}\tilde{\Sigma} \\
\bar{\omega} \\
\bar{\omega}\end{array}$ & $\mid \begin{array}{l}\hat{\Sigma} \\
\bar{\infty} \\
\bar{\infty}\end{array}$ & $\mid \begin{array}{l}\tilde{\Sigma} \\
\bar{\infty} \\
\bar{\omega}\end{array}$ & $\mid \begin{array}{l}\hat{\Sigma} \\
\vec{\omega}\end{array}$ & $\mid \begin{array}{l}\tilde{\Sigma} \\
\vec{\omega} \\
\vec{\omega}\end{array}$ & $\mid \begin{array}{l}\hat{\Sigma} \\
\bar{\infty} \\
\bar{\infty}\end{array}$ & $\mid \begin{array}{l}\hat{\Sigma} \\
\vec{\omega}\end{array}$ & $\mid \begin{array}{l}\hat{\Sigma} \\
\tilde{\omega} \\
\bar{\infty}\end{array}$ & $\mid \begin{array}{l}\hat{\Sigma} \\
\vec{\omega}\end{array}$ & $\frac{\hat{n}}{\Sigma}$ & $\begin{array}{l}\sum_{\Sigma}^{n} \\
\vec{\omega}\end{array}$ & $\mid \begin{array}{l}\tilde{\Sigma} \\
\tilde{\omega} \\
\bar{\infty}\end{array}$ & 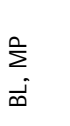 & $\vec{\infty}$ \\
\hline 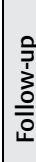 & & 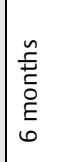 & 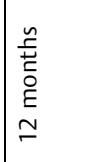 & 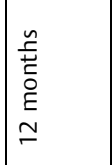 & 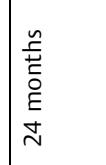 & 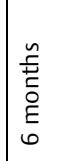 & 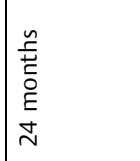 & 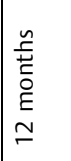 & 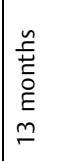 & 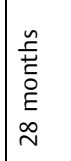 & 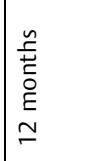 & 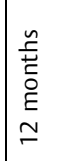 & 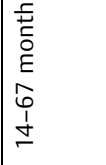 & 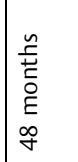 & 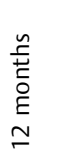 & 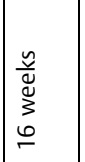 & $\begin{array}{l}\text { ñ } \\
\text { 产 } \\
\text { E } \\
\text { E } \\
\text { m. }\end{array}$ & 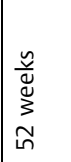 \\
\hline = & & in & $\infty$ & $\nabla$ & in & - & 8 & $F$ & $\sigma$ & $\infty$ & - & $\stackrel{\Perp}{\llcorner}$ & $=$ & - & 0 & 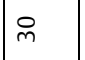 & - & $\stackrel{\Perp}{\sim}$ \\
\hline 豆 & & $\underset{\simeq}{\Xi}$ & $\tilde{0}$ & $\overrightarrow{0}$ & 0 & 只 & $\underset{\longleftarrow}{\check{L}}$ & ठ & 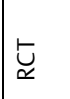 & $\underset{\simeq}{\Xi}$ & 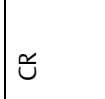 & 0 & 0 & 芩 & o & 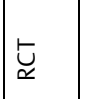 & ऽ & $\approx$ \\
\hline & 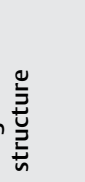 & U్ & $\breve{\sim}$ & $\breve{\sim}$ & ज্র & 顸 & $\breve{\sim}$ & $\ddot{n}$ & 约 & $\breve{u}^{\prime}$ & $\frac{\breve{s}}{z}$ & 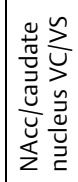 & 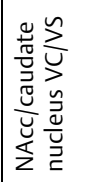 & 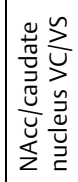 & 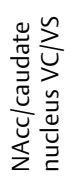 & 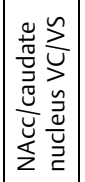 & 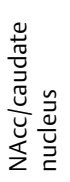 & $\frac{U}{\bar{J}}$ \\
\hline
\end{tabular}




\begin{tabular}{|c|c|c|c|c|c|c|c|c|c|c|c|c|c|}
\hline \multicolumn{2}{|c|}{ 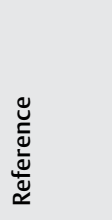 } & 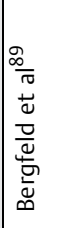 & 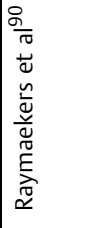 & 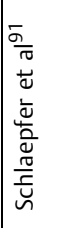 & 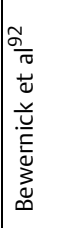 & 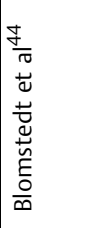 & 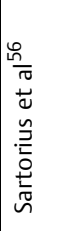 & 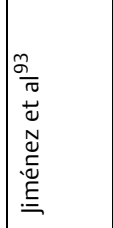 & 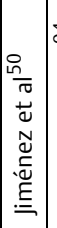 & 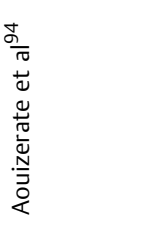 & 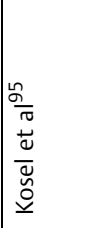 & 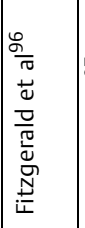 & 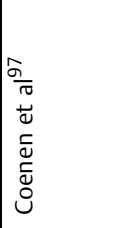 \\
\hline \multicolumn{2}{|c|}{ 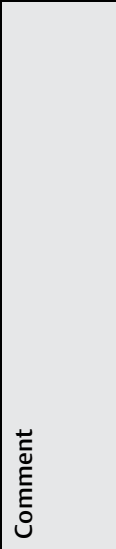 } & 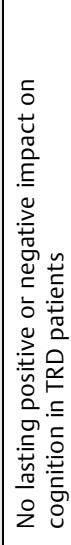 & 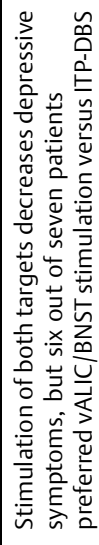 & 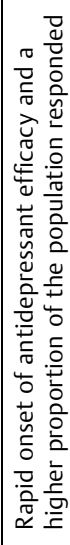 & 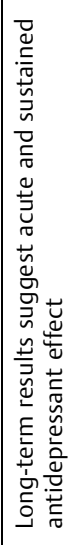 & 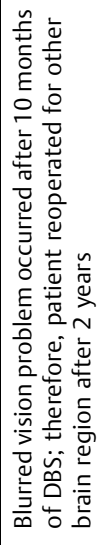 & 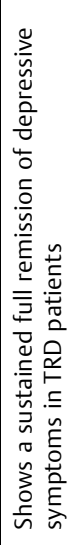 & 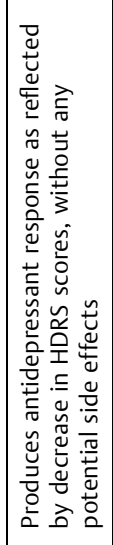 & 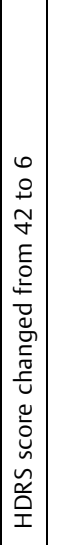 & 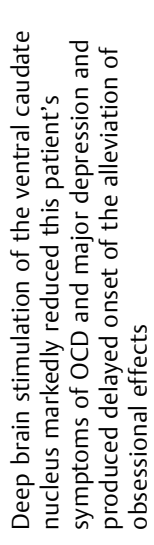 & 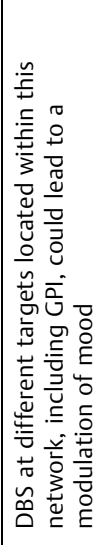 & 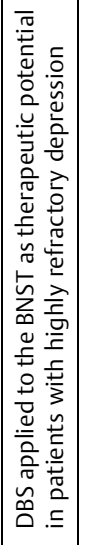 & 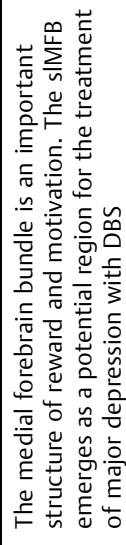 \\
\hline \multirow{2}{*}{ 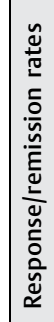 } & 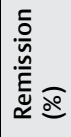 & $\stackrel{\leftrightarrow}{\grave{N}}$ & $\begin{array}{l}\stackrel{\sim}{\infty} \\
\infty \\
\sim\end{array}$ & in & in & $\Sigma$ & $\frac{\pi}{z}$ & ○ & 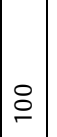 & $\Sigma$ & $\Sigma$ & $\Sigma$ & in \\
\hline & 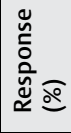 & 字 & $\frac{\vec{x}}{\stackrel{r}{r}}$ & $\infty$ & 요 & $\Sigma$ & $\frac{\pi}{z}$ & $\mathbb{z}$ & $\begin{array}{l}\tilde{R} \\
\dot{\omega} \\
\infty\end{array}$ & $\underline{z}$ & $\Sigma$ & $\Sigma$ & $\stackrel{\infty}{\infty}$ \\
\hline \multirow{4}{*}{ 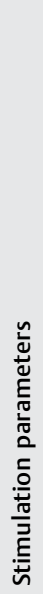 } & 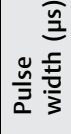 & ৪) & $\bar{z}$ & 8 & 8 & 8 & $\Sigma$ & 号 & 阜 & $\begin{array}{l}\stackrel{2}{1} \\
\frac{1}{\grave{a}}\end{array}$ & \& & 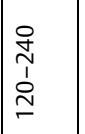 & $\Sigma$ \\
\hline & 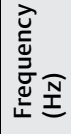 & $\begin{array}{l}\infty \\
\infty \\
1 \\
\tilde{n} \\
\end{array}$ & $\frac{\pi}{z}$ & 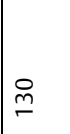 & 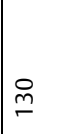 & $\stackrel{\circ}{m}$ & $\frac{\pi}{z}$ & 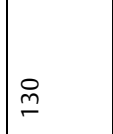 & $\stackrel{\circ}{m}$ & $\stackrel{\circ}{m}$ & 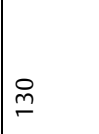 & 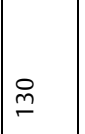 & $\Sigma$ \\
\hline & \multirow{2}{*}{ 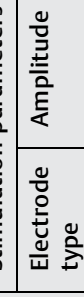 } & $\begin{array}{l}> \\
0 \\
\dot{j} \\
1 \\
\stackrel{1}{n} \\
\sim\end{array}$ & 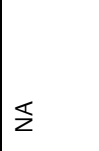 & $\begin{array}{l}> \\
0 \\
\dot{j} \\
\dot{j} \\
\dot{\sim}\end{array}$ & $\begin{array}{l}> \\
0 \\
\dot{p} \\
\dot{d} \\
\dot{\sim}\end{array}$ & $\begin{array}{l}\vec{a} \\
0 \\
\dot{j} \\
\infty \\
\stackrel{\sim}{u}\end{array}$ & $\begin{array}{l}2 \\
\text { nn } \\
0 \\
0\end{array}$ & $\begin{array}{l}> \\
0 \\
0 \\
\dot{1} \\
0 \\
\dot{m}\end{array}$ & $\begin{array}{l}2 \\
0 \\
\dot{b} \\
0 \\
0 \\
\dot{m}\end{array} \mid$ & $\begin{array}{l}> \\
0 \\
\dot{+} \\
\dot{1} \\
\dot{i}\end{array}$ & $\begin{array}{l}> \\
\infty \\
\infty \\
\dot{1} \\
\omega \\
m \\
m\end{array}$ & 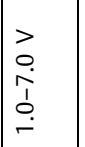 & $\Sigma$ \\
\hline & & $\vec{\infty}$ & $\vec{\infty}$ & $\begin{array}{l}\hat{\infty} \\
\vec{\infty} \\
\vec{\infty}\end{array}$ & $\begin{array}{l}\hat{\infty} \\
\vec{\infty}\end{array}$ & $\begin{array}{l}\hat{\infty} \\
\vec{\infty}\end{array}$ & $\frac{\pi}{z}$ & 㐫 & क & $\vec{\infty}$ & $\mid \begin{array}{l}\tilde{\Sigma} \\
\dot{\omega} \\
\dot{\omega}\end{array}$ & $\mid \begin{array}{l}a \\
\Sigma \\
\dot{\omega}\end{array}$ & $\vec{\infty}$ \\
\hline \multicolumn{2}{|c|}{ 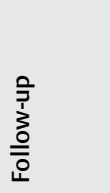 } & 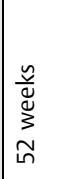 & 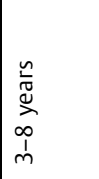 & 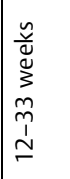 & 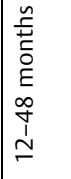 & 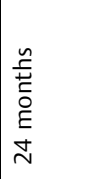 & 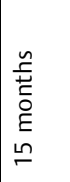 & 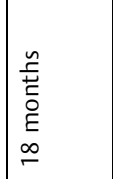 & 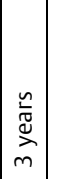 & 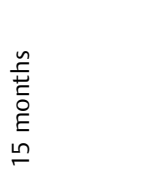 & 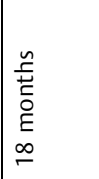 & 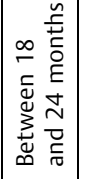 & 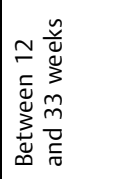 \\
\hline \multicolumn{2}{|c|}{$=$} & $\stackrel{\mathscr{N}}{\sim}$ & h & $\wedge$ & $\infty$ & - & - & - & - & - & - & in & $\stackrel{\sim}{\sim}$ \\
\hline \multicolumn{2}{|c|}{ 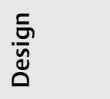 } & 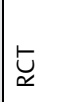 & $\tilde{0}$ & $\tilde{o}$ & $\tilde{0}$ & $\stackrel{\text { S }}{ }$ & ธ & 品 & $\stackrel{\sim}{\sim}$ & ษ & $\stackrel{\sim}{\cup}$ & $\tilde{\partial}$ & $\tilde{0}$ \\
\hline \multicolumn{2}{|c|}{ 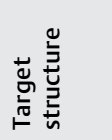 } & $\frac{v}{\bar{y}}$ & 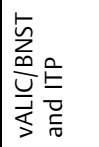 & 岕 & $\frac{\omega}{\Sigma}$ & $\begin{array}{l}\frac{5}{2} \\
\frac{0}{00} \\
\frac{0}{2}\end{array}$ & 全 & 徥 & $\hat{E}$ & 5 & $\overline{\mathrm{v}}$ & $\sum_{\infty}^{5}$ & $\sum_{u}^{u}$ \\
\hline
\end{tabular}

总离焉

产

오응

㻤 in

贾至今

至

竎

辗

은 喜 节

a

نิ

要 $\frac{0}{\frac{0}{2}}$

$\stackrel{\Xi}{E}$

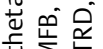

एٓن

م.

进总

ธิ

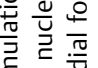

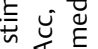

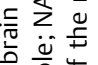

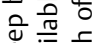

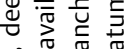
जै

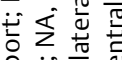

는

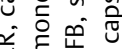

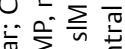

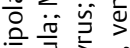

के ए

in 0

중

空

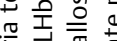

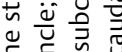

㽞

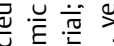

政

这 艺这 。

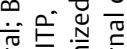

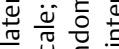
可

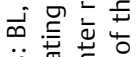
谖察 든 흑 ऽे 京苍蓄 
DBS, neurofunctional surgery, electrode implantation, neurostimulation, neuromodulation, and psychiatric surgery, and their equivalents in Portuguese, Spanish, German, French, Dutch, and Czech. The terms were searched in all fields of the databases.

For the selection of the studies, the following inclusion criteria were adopted: 1 . studies correlating TRD and DBS as a treatment strategy; 2. studies published until September 2018; 3. publications in English, Portuguese, Spanish, German, French, Dutch, and Czech. 4. human experimental trials.

The exclusion criteria chosen for the present review were: 1. duplicate publications; 2 . studies involving animals; 3. studies involving the ethical and existential implications of DBS; 4. editorials, comments of the authors, and debates; 5. studies addressing depression secondary to any other diseases; 6. studies reporting any neurosurgical interventions other than DBS.

Articles with short- and long-term outcomes of the same trial published separately were both included, because some featured new patients and/or novel stimulation strategies. Official documents released by relevant societies and references used in experimental articles were also examined. Subsequently, the results were manually reviewed and selected for analysis.

\section{Results}

A total of 46 papers were selected for the present systematic review, including 9 multicenter randomized controlled trials (RCTs), 19 open label studies, and 18 case reports (-Fig. 1, - Table 1). The targets employed were the BNST, the GPI, the ITP, the LHb, the MFB, the NAcc, the SCg, the sIMFB, the vALIC, the ventral caudate nucleus (VCN), and the VC/VS.

A double-blind multisite RCT (20 institutions), known as the Brodmann Area 25 Deep Brain Neuromodulation (BROADEN) trial, targeting the SCg and involving 90 participants, has been the largest psychiatric DBS study so far. Response to treatment was defined as a decrease $\geq 40 \%$ in the Montgomery-Åsberg Depression Rating Scale (MADRS) score from baseline and no worsening in the Global Assessment of Functioning (GAF) score. The DBS parameters were adjusted using an algorithm, and the concomitant use of ADs was allowed as long as the doses remained steady. Patients with chronic, unremitting depression were implanted and randomly assigned to 6 months of active or sham DBS, followed by 6 months of open-label SCg DBS. Both groups exhibited overall improvement on daily function (average of $132.2 \%$ ), $92 \%$ of the patients reached a MADRS decrease from baseline of at least $50 \%$, and $58 \%$ of them had complete remission. $^{78}$

However, during the double-blind sham-controlled phase (12 patients with active versus 5 with sham DBS), the sham response rate was $17 \%$, but no statistically significant difference was found in the responses of both groups. No psychiatric or neuropsychological adverse events (AEs) were reported at the 6- or 12-month follow-ups. Major AEs included suicidality (3/17), with 2 suicides in the control group during the 6-month open-label phase, anxiety (5/17), infection (5/17), system malfunction (3/17), and worsening of the depression $(2 / 17){ }^{78}$

During the long-term open-label follow-up at 12,18 , and 24 months, the responses were $29 \%, 53 \%$, and $49 \%$, respectively. Of the 30 subjects in this phase, 26 decided to continue with DBS stimulation. A futility analysis was performed when approximately half of the patients received active DBS, completing the double-blind phase, indicating that the study had a $17 \%$ chance of success if continued. Although at the given timepoint this number did not meet the definition for futility ( $\geq 10 \%$ chance of success), the study was halted. $^{78}$

The rationale of targeting the SCg started in a pioneer study that included six patients aiming to access the feasibility and safety of DBS modulation of the SCg and of the adjacent white matter. A decrease $\geq 50 \%$ in the 17 -item Hamilton Depression Rating Scale (HDRS-17) was considered a response to treatment. Response and remission rates at the 1 - and 6-month follow-ups were $35 \%, 10 \%, 60 \%$, and $35 \%$, respectively. ${ }^{26}$

Neuropsychological analyses revealed that DBS was also effective to improve self-negative bias. ${ }^{71}$ Neuroimaging evidenced metabolic changes, and a neurocognitive assessment in six patients proved the procedure to be safe. ${ }^{58}$

A 12-month follow-up incorporated an additional 14 patients to this cohort, and adjustable stimulation parameters were based on the presence of acute behavioral effects. The benefits were maintained and no permanent AEs occurred. ${ }^{57}$ The extended follow-up showed average response rates of $62.5 \%, 46.2 \%, 75 \%$, and $64.3 \%$ after $1,2,3$, and 3 to 6 years, respectively. Overall, AEs were transient, and the most frequent was suicidality (3/20), with a confirmed suicide at 35 months and an attempted suicide at 75 months. Also, worsening of the depression (3/20), infection (3/20), and 1 case of perioperative seizure were registered. ${ }^{63}$ Despite the initial good response, 1 patient from this series relapsed 4 years later; nonetheless, the use of tranylcypromine, a monoamine oxidase inhibitor (MAOI), along with DBS decreased the MADRS score by $60 \%$ after 4 months. ${ }^{67}$

Another case study from this series presented transient oscillation of response and important depressive episodes, one of them related to battery depletion. However, the patient was responsive to medication adjustments and obtained an overall sustained response. ${ }^{62}$

Investigators replicated the design of SCg DBS in a multicenter approach involving 21 patients during 12 months, but employing a different stimulation device. Setting the response criterium at $\geq 50 \%$ decrease in the HDRS- 17 , the results were $57 \%, 48 \%$, and $29 \%$ at the 1 -, 6 -, and 12 -month follow-ups, respectively. After 12 months of DBS, establishing the response criterium at $\geq 40 \%$, total responders increased to $62 \%$, which was attributed to amelioration in disease severity. Major AEs were nausea/vomiting and suicidality (2/21). ${ }^{68}$

The same system was also investigated in a cohort study with a sham-controlled design including 10 unipolar TRD individuals and 7 bipolar subtype II treatment-resistant 
patients. At the 2-year endpoint, the response was $92 \%$, whereas the remission was 58\%. Two suicide attempts were reported, an MDD remitter at 2 years, and a bipolar patient at 54 months of follow-up. ${ }^{64}$

In a pilot study, baseline frontal theta cordance (FTC) appeared as a biomarker for predicting 6-month clinical response to SCg DBS for TRD. In addition to that, lower FTC at baseline and higher FTC after 4 weeks were predictors of lower depression severity scores at the 24-week follow-up. ${ }^{65}$

A multicenter double-blind randomized crossover of 13 months was carried out with 9 MDD patients resistant to treatment to evaluate the effects of high $(130 \mathrm{~Hz})$ vs low $(20 \mathrm{~Hz}$ ) frequency BA 25 DBS. Response ( $\geq 40 \%$ MADRS) was achieved by $4 / 9$ patients, with similar improvements in high and low frequency stimulation groups after 6 months. In the second period of the trial, the high frequency group showed higher improvement regarding the response criteria. $^{80}$

An uncontrolled double-blind (delayed versus nondelayed stimulation onset) study included five patients with TRD and one with bipolar affective disorder type I who underwent SCg DBS. Two attained remission (HDRS$24 \geq 10$ ) at 24 and 36 months, with no AEs due to acute high intensity stimulation $(>10 \mathrm{~V})$. The main outcome was depression severity assessed using the HDRS-24, and the secondary outcome parameters were MADRS and Beck Depression Inventory (BDI) scores. Acute 24-hour stimulation caused moderate decreases in all the scales. Between 24 and 36 weeks, 2 patients were remitters and 4 were nonresponders. $^{69}$

Another report by the same group included participants in the aforementioned cohort, encompassing seven patients with TRD and one with bipolar affective disorder type I. The response rate was $51 \%$, and 2 patients achieved remission (33.3\%) at the 28-month and 4-year follow-ups. No statistical differences were found between different onset groups. ${ }^{81}$

A diffusion tensor imaging study on this same series found that the only responder had the contacts located bilaterally in the posterior gyrus rectus (BA 14). This displayed strong connectivity between the stimulated regions and the mPFC. $^{76}$

A Spanish group initiated a study in 2008 performing SCg DBS in 8 TRD patients following an open-label design. ${ }^{66}$ In a preliminary result, 1 patient from this series relapsed at 4 months and presented with psychotic symptoms. The DBS system was turned off and, after nine sessions of frontal ECT, when DBS was turned on again, the patient successfully reached remission. ${ }^{60}$

After 1 year of stimulation, they obtained a response of $62.5 \%$ in the HDRS -17 and remission in $50 \%$ of the cases, with improvement in social function and neurocognitive safety, as well as benefit for the memory. ${ }^{74}$ Except for a suicide attempt in the group of nonresponder patients, no other serious AEs occurred.

Subsequently, stimulation was ceased in the 5 previous responders under a double-blind randomized design, resulting in sustained remission (2/5), relapse (2/5), and progressive worsening without relapse $(1 / 5)$ in their 3 -month sham protocol. ${ }^{73}$ Simultaneously, remitters underwent doubleblind sham stimulation. ${ }^{72}$ Fluorine-18-fluorodeoxyglucose $\left({ }^{18} \mathrm{~F}-\mathrm{FDG}\right)$ positron emission tomography (PET) scans evidenced metabolism decreases in the dACC (BA 24), in the premotor region (BA 6), and in the putamen, not followed by changes in HDRS-17 scores.

In a pilot cohort, four patients with TRD underwent $\mathrm{SCg}$ DBS surgical procedure. After that, the frequency and pulse widths were randomly changed weekly. Evaluations of changes in mood and depression were performed using a visual analogue scale (VAS) and the HDRS-17. Longer pulse widths (270-450 $\mu \mathrm{s})$ were related to short-term clinical improvement (HDRS-17) in 3 participants and to positive mood response (according to results indicated in the VAS) in all of the patients. No associations between DBS frequency and mood or clinical response were found. After 6 months of the open-label postoptimization phase, 2 patients (50\%) showed clinical response, and 1 showed partial response. ${ }^{70}$

The same group of researchers carried out a double-blind trial including the same patients. They found that the stimulation was related to decreases in serum BDNF concentration compared to pre-DBS baseline. ${ }^{75}$

In an open-label cohort of 11 patients, the fibers activated were proven to be more related to the response than the site of the implanting (mainly projections to BA 10), using whole brain activation volume tractography. At 6 months, 8 patients had an increase in current from 6 to $8 \mathrm{~mA}$. Response, considered as $\geq 50 \%$ decrease in the HDRS-17, was reached by $72.7 \%$ of the sample at 6 months, and by $81.8 \%$ at 12 months. Remission criterium (HDRS-17 $\geq 7$ ) was attained by 6 patients, and 2 never met it. One of them had minimal variation in the HDRS-17, whereas the other achieved $40 \%$ decrease in this score at 12 months. A whole brain activation volume tractography and the common probabilistic tract map generated for all subjects (responders and nonresponders) at 6 months featured the inclusion of the forceps minor, the uncinate fasciculus, the frontostriatal fibers, and of the cingulum bundle. ${ }^{79}$

In an Argentinian case report, patient-blind unilateral stimulation produced rapid mood worsening on the left hemisphere. Most electrodes placed in the SCg and in the adjacent white matter produced stimulation related to acute onset of orthostatic hypotension, both at the postoperative testing and at a the 6-month assessment (the contacts were permanently kept turned off). No alterations were observed in the opposite hemisphere. ${ }^{61}$

In a French case report, a patient with long-term MDD and TRD, who had undergone extensive unilateral ECT that led to cognitive deficits, presented with late postoperative seizures as a possible side effect, displayed at standard stimulation parameters ( $90 \mu \mathrm{s}, 130 \mathrm{~Hz}, 4.2 \mathrm{~V}$ ). Most likely, DBS has revealed a previously existing temporal lobe epilepsy, although the participant had no individual or family history of convulsions. The patient was responsive to treatment. ${ }^{77}$

A study from England reported a patient with bipolar disorder and treatment resistance, with an infarct in the right thalamus (dorsomedial nucleus) that produced severe depressive symptoms within hours and TRD at 9 months 
(BDI-II: 41; Beck Anxiety Inventory: 26). DBS of sACC at high frequency $(>150 \mathrm{~Hz})$ did not produce any clinical effects, probably due to the reduction in structural connectivity from the sACC back to the amygdala on the right side. Projection to frontal areas was not clearly differentially disrupted. The patient was followed-up 1 year after the procedure, when the battery of the DBS was running low, but cessation of stimulation had no effect. This patient died in his sleep 16 months after the DBS surgery. ${ }^{59}$

In a randomized clinical trial, 25 patients in the Netherlands underwent DBS of the vALIC for TRD. An open-label optimization trial was conducted for 52 weeks followed by a sham-controlled double-blind multisite crossover RCT. The response criterium was $\geq 50 \%$ decrease in the MADRS from baseline to the $16^{\text {th }}$ week of the blind phase, while the remission criterium was HDRS-17 $\geq 7$ at the $2^{\text {nd }}$ assessment. At the end of the optimization phase, 10 patients were responders and 15 , non-responders. In the crossover phase, 16 of these patients - 9 responders and 7 nonresponders - participated. During active DBS, the HDRS-17 scores were significantly lower (13.6). Adverse events included: suicide attempts (5), increased suicidal ideation (2), suicide (1), euthanasia (1), and surgery-related extreme nausea (1) that interrupted the operation, which was performed 2 weeks later with success. Battery depletion was suspected in two patients. Active DBS had significant antidepressant effect in 10 out of 25 TRD patients compared with sham DBS, classified as responders ( $\geq 50 \%$ decrease) and partial responders ( $\geq 25$ but $<50 \%$ decrease) ${ }^{88}$ No permanent impact (either positive or negative) on cognition was observed in a posterior study with the same sample. ${ }^{89}$

A double-blind crossover trial with seven TRD patients investigated the stimulation in either the anterior limb of the internal capsule/BNST or in the ITP. All of the patients participated in the follow-ups for at least 3 years, but some were followed-up up to 8 years after the procedure. A significant average decrease in the HDRS-17 score (61\%) was attained by 5 responders and 2 remitters. Only one participant preferred ITP stimulation. Most patients reported fluctuant worsening of depressive symptoms and suicide ideation, and the patient that preferred ITP stimulation presented with transient extrapyramidal-like AEs (hypomimia, micrographia, hesitant walking, and less fluent movement). Two patients had a suicide attempt history prior to implanting and committed suicide at 39 and 80 months after the procedure, respectively. ${ }^{90}$

A 16-week randomized blind sham-controlled trial of DBS, known as RECLAIMTM, targeted the VC/VS in 30 patients with TRD, with a subsequent open-label phase. The response, set as a decrease $\geq 50 \%$ in the MADRS score, was $20 \%, 26.7 \%$, and $23.3 \%$ at the $12-, 18$-, and $24-$ month follow-ups, respectively. However, no significant differences in response rates were found between the active and sham treatments, or changes in the MADRS scores at the end of the 16-week controlled phase. A total of 71 serious AEs were recorded for 22 patients, and the most frequent were worsening of the depression (8), suicidal ideation (5 in the active and 3 in the sham group), suicide attempts (4), and a completed suicide of a nonresponder who ceased stimulation while preparing for explanting. During the blind phase, the most frequent psychiatric AEs in the active group were worsening depression and insomnia. ${ }^{10}$

In a multisite open-label investigation, 14 MDD patients and 1 bipolar (subtype not specified), 13 of which had failed both $\mathrm{AD}$ and ECT, and 2 who were also resistant to vagus nerve stimulation, were treated with DBS of the VC/VS. The response rates were $40 \%$ and $53.3 \%$ in the HDRS, and $46.7 \%$ and $53.3 \%$ in the MADRS, and the remission rates were $20 \%$ and $40 \%$ in the HDRS, and $26.6 \%$ and $33.3 \%$ in the MADRS at the 6-month and last follow-ups, respectively. ${ }^{83}$

A following study enrolled two additional patients, both AD- and ECT-resistant, who also underwent DBS of the VC/ VS. Response was attained by 53 and $71 \%$ of the sample $(n=17)$ at the 3-month and last follow-ups, respectively. Interestingly, $35 \%$ of the patients continued in remission (MADRS score $\leq 10$ ) at the last follow-up, and a remarkable reduction in suicidality occurred at 1 month and persisted in the next 12 months $(p \geq 0.001)$. Serious AEs related to DBS included: anxiety, autonomic effects, mood changes, and paresthesia. However, they were transient and, after adjusting the stimulation parameters, all of them disappeared. $^{84}$

One of the patients of the aforementioned multisite openlabel investigation, ${ }^{83}$ who had been a remitter for 4 years, experienced increase in smoking (50-200\%) and concurrent worsening depressive symptoms in 3 different occasions, all related to interruption of DBS caused by battery depletion. Nevertheless, once DBS stimulation was restarted, the smoking pattern reverted to baseline and the depressive symptoms decreased. ${ }^{85}$

A patient with TRD, comorbid bulimia, and borderline personality disorder showed improvement in depression (as per results in the HDRS score) after initial placement of electrodes in the ITP without electrical stimulation, probably because of a microlesion effect. After a phase of stimulation $(130 \mathrm{~Hz}, 0.45 \mu \mathrm{s}, 2.5 \mathrm{~V})$, it was discontinued in a double-blind fashion, and the HDRS score did not return to preoperative levels, remaining between 2 and 8 . This patient was later explanted and remained in remission up to 7 years. ${ }^{93}$

A patient with a history of treatment-resistant OCD, recurrent MDD, and unsuccessful cognitive-behavioral therapy was referred to DBS of the VCN. Nonetheless, several AD strategies improved the depressive symptoms prior to the procedure. In the first 3 months of stimulation, depressive symptoms progressively worsened, but at the 6-month follow-up, the patient achieved MDD remission (HDRS $=7$; Hamilton Anxiety Rating Scale $=10$ ), which was sustained until the end point, 15 months after the surgery. The patient also attained OCD remission, but more slowly, markedly between the 12- and 15-month follow-up, with progressive increase in Global Assessment of Functioning (GAF) scores. No negative neuropsychological effects were noted. ${ }^{94}$

A group of 10 patients presenting with very severe forms of TRD, refractory to ADs, psychotherapy, and ECT, underwent DBS of the NAcc. Response (50\% decrease in the HDRS28 ) was reached by $50 \%$ of the patients at the 12 -month 
follow-up, and 3 participants achieved remission (HDRS$28 \leq 10$ ) for a period of 1 month. ${ }^{98}$

The long-term effects of DBS of the NAcc were assessed in the same group of participants described above ${ }^{98,99}$ and in an additional patient enrolled posteriorly. Follow-ups were carried out 12 months, 24 months, and 4 years after the procedure with 11,10 , and 5 patients, respectively. Adverse events related to DBS were transient. By the 12-month follow-up, 1 patient had committed suicide and 1 had attempted suicide, both nonresponders to the surgery. After 12 months, $45 \%$ of the participants were considered responders, and did not show worsening symptoms at the 4year follow-up. ${ }^{100}$

In a double-blind placebo-controlled trial, three patients with extreme forms of TRD (resistant to psychotherapy, ADs, and ECT) received DBS implantation in the NAcc. The voltage ranged from 0 to $4 \mathrm{~V}$ in $1-\mathrm{V}$ steps, in a double-blind manner. At each step, HDRS-24 and MADRS were reapplied, and a negative correlation was observed for both scores in all of the patients. No relevant AEs occurred. Single items of both scales, often used to assess aspects of anhedonia, were verified, but no significant changes were found, in spite of clear clinical changes in anhedonia. Metabolic imaging displayed activations in bilateral VS (including NAcc), bilateral DLPFC and DMPFC, bilateral cingulate cortex, and bilateral amygdala, simultaneously with deactivations in the vmPFC, the ventrolateral PFC, the dorsal caudate nucleus, and in the thalamus. ${ }^{43}$

In a case report of a patient with a 20-year history of MDD, agoraphobia, and alcohol dependence for the previous 10 years, DBS of the NAcc produced acute pleasure. In 12 months, the patient became an occasional drinker. However, decreases in depression or anxiety were minimal. ${ }^{82}$

A depressed woman, with a 46-year history of severe MDD and 9 years of TRD, failed to respond to ADs and ECT. Her depressive episode at the intervention included delusions of guilt, mutism, and pronounced anxiety, with HDRS-21 rates around 45 . Deep brain stimulation of the LHb produced full remission of depressive symptoms within a period of 4 months. The patient relapsed, and the voltage was increased, leading to stable remission. One accidental switch off caused an additional relapse, but it was transient. ${ }^{56}$

The superolateral branch of the medial forebrain bundle (SIMFB) was targeted based on a neuroanatomical and functional hypothesis using new fiber tracking techniques: two opposing systems, the ATR and the sIMFB, were anatomically described and assumed to mediate negative (ATR) and positive (sIMFB) emotions. ${ }^{48}$

A decrease $\geq 50 \%$ in the HDRS was achieved by 6 patients, and 4 reached remission 12 months after DBS of the slMFB. Moreover, long-stable effects were reported up to 4 years after the procedure. The main AEs were oculomotor effects (blurred and double vision), responsive to reduction of amplitude of stimulation. Discontinuation of a nonresponder at 18 months decreased the score, but not exceeding baseline, and explantation kept remission until 12 months. ${ }^{92}$

The same design used above was replicated by the Houston group using deterministic tractography. After 52 weeks,
4 out of the 5 remaining patients that ended the trial had a decrease $>70 \%$ in the MADRS scores compared to baseline. The modulated fiber tracts revealed significant common orbitofrontal connectivity in all of the responders. Neuropsychological testing verified safety, and ${ }^{18} \mathrm{~F}$-FDG-PET cerebral metabolism evaluations at baseline and at 52 weeks showed minimal changes. Increased depression was associated with battery depletion in four patients, and accidental deactivation in three. ${ }^{101}$

Evaluation of the tractographies showed that responders typically have their active contacts exclusively situated in the center of the triangle, with no contact with the nuclear environment. Thus, every treatment should be based on individual slMFB (tractography) geometry. ${ }^{97}$

A case report presented a patient with TRD and nervous anorexia who was treated with DBS and showed great response. However, after 10 months, she presented blurred vision and was reoperated with electrodes placed on the BNST. At 12 months, the results were: MADRS $=13$; HDRS $=6$; $\mathrm{HAM}-\mathrm{A}=5 .{ }^{44}$

A pilot open-label series included five female patients resistant to AD and ECT, who underwent DBS of BNST. Clinical response was observed by means of various assessments rather than by a stated definition. Stimulation induced: 1 remission at 6 months; 1 response and 1 remission at 12 months; 3 remissions at the last follow-up, 2 of them stable (MDRS of 1 and 3 ) up to 6 years; and an eventual reoccurrence and restoring of remission after battery replacement. One patient had explantation of DBS, which was reimplanted in the SACC, but, by the end of the second treatment, she committed suicide. A significant increase in quality of life and depression scores, as well as neurocognitive stability, were attained. Two suicide attempts, apparently not related to stimulation, occurred during the trial, and one of these patients reached remission later. Transient insomnia was the most common AE related to increase in stimulation. ${ }^{96}$

An anecdotal case report targeted the GPI for TRD and severe tardive dyskinesia (TD) in a patient with a history of failing to over 60 psychotropic drugs, who had been treated with typical and atypical neuroleptics, and developed severe neuroleptic-induced TD. The patient attained $a \geq 50 \%$ decrease in the HDRS 18 months after DBS implantation. The HDRS score dropped from 26 at baseline preoperatively to 13 at the 18-month follow-up, whereas the Burke-Fahn-Marsden Dystonia Rating Scale score decreased from 27 to 17.5 (35\%). ${ }^{95}$

A preliminary study of four patients targeted the NAcc and, in the event of failure, the caudate nucleus, in a limbic vs cognitive fashion. The primary and secondary outcomes were $\geq 50 \%$ HDRS and remission, defined as HDRS $=7$ after 4 months, respectively. Stimulation of the NAcc was performed from the $1^{\text {st }}$ to the $5^{\text {th }}$ month. At month 5 , nonresponders underwent stimulation of the caudate target until month 9 , followed by a 6 -month extension phase (up to month 15), with adaptable parameters and concomitant treatments. A significant improvement in mood was achieved by 3 patients, with lower HDRS scores at the end 
of the 15 months. Following the start of stimulation, benefit was obtained at the extension phase, with open parameters. One patient did not meet response criteria at month 5 , but NAcc stimulation was kept due to clinical perception of improvement. Furthermore, aripiprazole was added at month 11 , leading the patient to a stable improvement until reaching response. ${ }^{86}$

A case report featured amplitude and dynamics of the mood changes, systematically quantified using the HDRS-17, in a nonresponder after DBS of the Nacc. The patient rapidly achieved and sustained remission 11 months after increasing the voltage of the most distal contact of each electrode located in the NAcc to $5 \mathrm{~V}$. Some worsening due to battery depletion was also reported. ${ }^{87}$

\section{Discussion}

Deep brain stimulation research for the treatment of patients with TRD has been marked by amelioration ${ }^{102}$ contrasting with inconsistent results of the three largest multicenter RCTs. ${ }^{10,78,88}$ Therefore, it could be inferred that DBS is not effective for TRD, at least in the way it has been currently performed and assessed. Aiming to understand these controversial outcomes, we tried and dissected factors that may be impacting trials and leading to fails.

On the one hand, little can be said about the efficacy of SCg as a DBS target based exclusively on the interrupted BROADEN trial. ${ }^{78}$ On the other hand, open-label studies focused on optimizing targets, as well as on mapping response patterns, patient subtypes, and connectomics, obtaining exceptional results. $^{79}$

The largest study followed the standard paradigm focusing on the Food and Drug Administration (FDA) validation at 6 months, with restricted parameters, that is, the surgical intervention was adequately isolated by not allowing postsurgical support, psychological or pharmacological treatment before the trial, and potentially reduced the chance of patient recovery at the short endpoint of the futility analysis. $^{78}$

A significant increase in response after SCg DBS was observed in the open-label original series from the $1^{\text {st }}$ to the $3^{\text {rd }}$ year, since the average response rose from 62.5 to $75 \% .{ }^{62,63}$ In sum, BROADEN could have been more thorough in terms of duration, adjustment of parameters, and optimization phase.

Considering open-label studies and case reports, the $\mathrm{SCg}$ remains promising, although BA 24 is probably the key area underlying the effects. ${ }^{61}$ In addition to this, unilateral vs bilateral hemisphere stimulation matters persist. ${ }^{49}$

The blind-treatment phase of the RECLAIM ${ }^{\mathrm{TM}}$ trial was probably too short and avoided high stimulation parameters to preserve blinding and prevent AEs. No significant differences were found during the sham phase, contrasting with the findings of a previous phase of the trial, in which $36 \%$ of the patients achieved response in 1 year and $92 \%$ in 2 years. ${ }^{64}$

The solely good performance of the vALIC large RCT highlights some particular characteristics, such as: a smaller sample; a 52-week open-label parameter optimization phase; stratification of response (partial response if 25$50 \%$ decrease in symptoms); and the intent-to-treat analyses to discriminate response from non-response. ${ }^{88}$

The case reports corroborated the severity of TRD in highly resistant patients and related complications such as $\mathrm{TD}^{95}$ and cognitive deficit after years of ECT. ${ }^{77}$ They also described strategies biased by the small casuistic, which were nevertheless life-changing in the context they were proposed, that is, contraindication to $\mathrm{ECT},{ }^{60} \mathrm{MAOI}$ restoring DBS response, ${ }^{67}$ and substance dependance. ${ }^{82}$ After all, these are common exclusion criteria in studies, but in the case reports selected, the patients presenting with them were treated using DBS.

Heterogeneity inherent to psychiatric neurosurgery occurs within trials in multiple domains: selected patients, pretrial treatments, trial designs (open label, crossover, and parallel), optimization of parameters (if allowed and duration), surgical technique, individual variability due to structural and functional connectivity, ${ }^{102}$ scales to define and monitor response and remission.

Major depressive disorder is a bureaucratic diagnosis, based on clinically-derived, however, arbitrary criteria. A mathematical analysis showed that 227 different combinations of depressive symptoms ${ }^{103}$ can fulfill the DSM-5 diagnostic criteria for MDD. ${ }^{21}$ Given that some items are multiple or alternative symptoms (i.e., insomnia or hypersomnia), if each component symptom is considered separately, 14,528 combinations are possible. $^{103}$

Lack of a global definition of TRD potentially adds a second level of phenotype heterogeneity labelled together with the population of interest; therefore, the inclusion criteria consistently diverge between studies. It is possible that by targeting DBS for TRD, distinct phenotypes/subtypes of this mental condition fall under the same label. This way, they have probably been addressed using the same circuits and the effects vary according to the deficits. Stage 5 treatment-resistant depression (irresponsiveness to three ADs and ECT) ${ }^{104}$ seems the most adequate definition of TRD for trial purposes.

The definition of response varies, but it is frequently set as a decrease by $50 \%$ in depressive symptoms assessed using HDRS and MADRS. ${ }^{105}$ Even though the former has different versions and numbers of items (i.e. HDRS-17, $-21,-24$, and -28 ), the exact scale is not always mentioned in the studies. ${ }^{44}$

The fallacy of thresholds, a methodological bias explored for AD trials with TRD patients, showed that scales lose statistical power when used to compare treatment against placebo. ${ }^{106}$ By doing so, researchers assume that sensibility and specificity are the same in both groups, responders in the placebo group might fit "intuitive definitions" of response less well than patients under treatment, and patients in the adjacencies of cutoff scores of scales are often clinically indistinguishable. $^{107}$

For Parkinson disease (PD), the Unified Parkinson's Disease Rating Scale ${ }^{108}$ was necessary to validate the evident impact DBS had on symptom control. Given that modeling mood disorders is even more complex, research on TRD should possibly follow the same path by developing a specific scale. $^{35}$ 
The MFB study showed exceptionally good immediate and sustained efficacy $(\sim 80 \%)$. This makes this target the most promising of the open-label trials selected. ${ }^{97}$

The MFB is the most rapid to produce response, most probably because it lies at the center of the reward pathway, ${ }^{14,91,109}$ with acute effects also more pronounced on the NAcc. ${ }^{87}$ Nonetheless, whenever acute responses are present, the insertional effect (possibly related to acute inflammatory mediators ${ }^{11}$ or glial released neurotransmitters ${ }^{12,32}$ in early time-points must be considered. Sustained and low progressive improvement in the blind stimulation cohorts and acute mood changes related to alterations in parameters months after surgery ${ }^{87}$ tend to indicate efficacy of the surgical procedure. ${ }^{110}$

Although sham designs mitigate placebo effect, especially if longer shams are employed, this effect is still relevant (five times stronger than medications in DBS for PD). It possibly happens due to expectation per se following the instructions of the doctor, follow-up visits, and high-frequency stimulation potentially rising subtle AEs and affecting patient blinding. Yet, placebo effect and spontaneous remissions are not usual in patients with very severe TRD. ${ }^{7,26}$ Worsening symptoms because of unintended "shams" such as battery depletion were frequently highlighted in the present systematic review, corroborating the efficacy of DBS. Strategies to overcome placebo effect include longer shams and optimization phases. Nevertheless, the latter may imply selection bias in the randomization phase. The counterpart effects, nocebo and lessebo, ${ }^{66,110}$ cannot be rejected whenever patients are aware of the possibility of being in the sham arm, which the inform consent provides.

The suicides reported appear to be dissociated from system malfunction or from changes in parameters, and were comparable to mortality rates in naturalistic studies. ${ }^{111,112}$ Whether suicide after DBS occurs due to lack of efficacy and disease progression or because stimulation lowers the suicide threshold remains unanswered.

Overall, other stimulation AEs are transient and responsive to parameter adjustment. Visual disturbances are particularly common in patients undergoing high stimulation parameters at some targets, especially the slMFB. Therefore, this $\mathrm{AE}$ is a relative limitation to sIMFB DBS. ${ }^{44}$ Emphasis should be given to investigational studies, as this target reportedly exhibits the most rapid and a sustained response. Additionally, high oculomotor-stimulating frequencies are likely associated with DBS efficacy. ${ }^{64}$

Optimal DBS parameter settings are still under debate. $^{31,34,53,75}$ Evidence points that short pulse width-low intensity, short pulse width-high intensity, as well as long pulse width-low intensity stimulation are the possible combinations. The high- versus low-frequency debate arises, ${ }^{31}$ with some strong evidences ${ }^{34,53}$ indicating that high-frequency stimulation promotes better $\mathrm{AD}$ response. ${ }^{73,80}$

The fact that the commercial value of being first to market is undoubtedly appealing ${ }^{25}$ might have contributed to the prematureness of the three pivotal researches. ${ }^{10,78,88}$ Whereas the trials herein presented have used open-loop systems, alternatively closed-loop or adaptive DBS systems, in dynamic stimulation settings based on a patient-control variable, in a feedback-like manner, tend to play a significant role in a near future. ${ }^{113}$ This dynamic model seems coherent with the most common symptoms of the disease and with the idea that different phenotypes fall under the umbrella of TRD.

Since in standard magnetic resonance imaging sequences the sIMFB is not visualized, tractography generates the hypothesis of a target, culminating in response above $80 \%$. Therefore, tractographies are mandatory for this target. ${ }^{97}$

Outcome predictors of efficacy of DBS for TRD appear to be related to symptoms rather than to the syndromic diagnosis, as underpinned by evidence of symptom-target relationship such as the connection of negative $\operatorname{mood}^{26}$ to the $\mathrm{SCg} 25$, the $\mathrm{MFB}$, and the NAcc. ${ }^{91}$ This brings psychiatrists to the operating room, where the presence of this professional enhances patient trust, ${ }^{28}$ and the functional neurosurgeon to a clinical interdisciplinary health care team. ${ }^{114}$

Evidently, treatment options for MDD have never been so diverse, and, yet, suicide and depression rates have been increasing. ${ }^{115}$ Deep brain stimulation is promising; however, it is restricted to specialized centers and highly selected patients, the market is dominated by a few companies, ${ }^{116}$ and the procedure is costly. ${ }^{117}$ This illustrates the long way ahead before DBS for TRD achieves efficacy and effectiveness.

Based on our exploratory exercise prior to the present systematic review of the literature, we conveniently conveyed inclusion criteria to allow psychiatric comorbidities, obtaining highly heterogeneous populations, closer to the reality of resistant populations. However, the theoretical modeling of DBS for TRD was compromised, posing a limitation to the present study. Furthermore, statistical analyses were not performed, since the trials selected are substantially different and, thus, not statistically comparable. Consequently, the successful and failing outcomes presented must be interpreted with caution, as these limiting factors potentially impair generalizations.

\section{Conclusion}

The current DBS research for TRD shed some light on the understanding of the most prevalent mental disorder. The studies here examined are among the most sophisticated to date. Nonetheless, they were not sufficient to reject or confirm the clinical pertinence of DBS. Despite the expansion of the therapeutic range of somatic therapies for depression, contemporary concerns on the repercussions of TRD and its lethality make DBS key to engross the list of treatment modalities. Thus, DBS remains one of the most promising and versatile strategies of this potential toolkit.

Conflicts of Interests

The authors have no conflicts of interests to declare.

\section{References}

1 Friedrich MJ. Depression is the leading cause of disability around the world. JAMA 2017;317(15):1517. Doi: 10.1001/jama.2017.3826

2 Kessler RC, Berglund P, Demler O, Jin R, Merikangas KR, Walters EE. Lifetime prevalence and age-of-onset distributions of DSM- 
IV disorders in the National Comorbidity Survey Replication. Arch Gen Psychiatry 2005;62(06):593-602. Doi: 10.1001/ archpsyc.62.6.593

3 Bergfeld IO, Mantione M, Figee M, Schuurman PR, Lok A, Denys D. Treatment-resistant depression and suicidality. J Affect Disord 2018;235:362-367. Doi: 10.1016/j.jad.2018.04.016

4 Fava M. Diagnosis and definition of treatment-resistant depression. Biol Psychiatry 2003;53(08):649-659. Doi: 10.1016/S00063223(03)00231-2

5 Leon AC, Keller MB, Warshaw MG, et al. Prospective study of fluoxetine treatment and suicidal behavior in affectively ill subjects. Am J Psychiatry 1999;156(02):195-201

6 World Health Organization. Depression. Geneva: World Health Organization, 22 March 2018. Available at: http://www.who.int/ news-room/fact-sheets/detail/depression. Access in: August 21, 2018

7 Kellner CH, Knapp RG, Petrides G, et al. Continuation electroconvulsive therapy vs pharmacotherapy for relapse prevention in major depression: a multisite study from the Consortium for Research in Electroconvulsive Therapy (CORE). Arch Gen Psychiatry 2006;63(12):1337-1344. Doi: 10.1001/archpsyc.63. 12.1337

8 UK ECT Review Group. Efficacy and safety of electroconvulsive therapy in depressive disorders: a systematic review and metaanalysis. Lancet 2003;361(9360):799-808. Doi: 10.1016/S01406736(03)12705-5

9 Ruhé HG, van Rooijen G, Spijker J, Peeters FPML, Schene AH. Staging methods for treatment resistant depression. A systematic review. J Affect Disord 2012;137(1-3):35-45. Doi: 10.1016/j. jad.2011.02.020

10 Dougherty DD, Rezai AR, Carpenter LL, et al. A randomized shamcontrolled trial of deep brain stimulation of the ventral capsule/ ventral striatum for chronic treatment-resistant depression. Biol Psychiatry 2015;78(04):240-248. Doi: 10.1016/j.biopsych. 2014. 11.023

11 Kringelbach ML, Jenkinson N, Owen SL, Aziz TZ. Translational principles of deep brain stimulation. Nat Rev Neurosci 2007;8 (08):623-635. Doi: 10.1038/nrn2196

12 Torres-Sanchez S, Perez-Caballero L, Berrocoso E. Cellular and molecular mechanisms triggered by Deep Brain Stimulation in depression: A preclinical and clinical approach. Prog Neuropsychopharmacol Biol Psychiatry 2017;73:1-10. Doi: 10.1016/j. pnpbp.2016.09.005

13 Lipsman N, Sankar T, Downar J, Kennedy SH, Lozano AM, Giacobbe P. Neuromodulation for treatment-refractory major depressive disorder. CMAJ 2014;186(01):33-39. Doi: 10.1503/cmaj.121317

14 Kurin DS. Trepanation in South-Central Peru during the early late intermediate period (ca. AD 1000-1250). Am J Phys Anthropol 2013;152(04):484-494. Doi: 10.1002/ajpa.22383

15 Zuccaro G. The dawn of neurosurgery in pre-conquest Mesoamerican territories. Childs Nerv Syst 2017;33(10):1621-1629. Doi: 10.1007/s00381-017-3464-4

16 Faulkner LA. Ancient medicine: sickness and health in Greece and Rome. 2nd ed. Michigan: Ichabod Press; 2015

17 Webster's Third New International Dictionary. Unabridged. 3rd ed. Springfield: Merriam-Webster; 2002. narc- or narco- combining form; p. 35

18 Ebbell B. The Papyrus Ebers: the greatest Egyptian medical document. Copenhagen: Levin \& Munksgaard; London: Oxford University Press; 1937

19 Leibowitz JO. Notes and Events. Plastic surgery. Electroshock therapy in Ibn-Sina's Canon. J Hist Med Allied Sci 1957;12(01): 71-72. Doi: 10.1093/jhmas/XII.1.71

20 Tsoucalas G, Karamanou M, Lymperi M, Gennimata V, Androutsos G. The "torpedo" effect in medicine. Int Marit Health 2014;65 (02):65-67. Doi: 10.5603/IMH.2014.0015

21 American Psychiatric Association. Diagnostic and Statistical Manual of Mental Disorders (DSM-5). 5th ed. Arlington: American Psychiatric Association; 2013
22 Ribot T. La psychologie des sentiments. Paris: Ancienne Librairie Germer Ballière et Cie; 1896

23 Vilela Filho O. Tratamento cirúrgico das desordens psiquiátricas. J Bras Neurocirurg 2009;20(03):362-364

24 Spiegel EA, Wycis HT, Marks M, Lee AJ. Stereotactic apparatus for operations on the human brain. Science 1947;106 (02754):349-350. Doi: 10.1126/science.106.2754.349

25 Bari AA, Mikell CB, Abosch A, et al. Charting the road forward in psychiatric neurosurgery: proceedings of the 2016 American Society for Stereotactic and Functional Neurosurgery workshop on neuromodulation for psychiatric disorders. J Neurol Neurosurg Psychiatry 2018;89(08):886-896. Doi: 10.1136/jnnp-2017317082

26 Mayberg HS, Lozano AM, Voon V, et al. Deep brain stimulation for treatment-resistant depression. Neuron 2005;45(05):651-660. Doi: 10.1016/j.neuron.2005.02.014

27 Mayberg HS, Riva-Posse P, Crowell AL. Deep Brain Stimulation for depression: keeping an eye on a moving target. JAMA Psychiatry 2016;73(05):439-440. Doi: 10.1001/jamapsychia try.2016.0173

28 Lipsman N, Giacobbe P, Lozano AM. Deep Brain Stimulation for the management of treatment-refractory major depressive disorder. In: Sun B, De Salles A, editors. Neurosugical treatments for psychiatric disorders. Dordrecht: Springer; 2015:95-104

29 Abosch A, Cosgrove GR. Biological basis for the surgical treatment of depression. Neurosurg Focus 2008;25(01):E2. Doi: 10.3171/FOC/2008/25/7/E2

30 Pandya M, Altinay M, Malone DA Jr, Anand A. Where in the brain is depression? Curr Psychiatry Rep 2012;14(06):634-642. Doi: 10.1007/s11920-012-0322-7

31 Udupa K, Chen R. The mechanisms of action of deep brain stimulation and ideas for the future development. Prog Neurobiol 2015;133:27-49. Doi: 10.1016/j.pneurobio.2015.08.001

32 Palazidou E. The neurobiology of depression. Br Med Bull 2012; 101:127-145. Doi: 10.1093/bmb/lds004

33 Price JL, Drevets WC. Neurocircuitry of mood disorders. Neuropsychopharmacology 2010;35(01):192-216. Doi: 10.1038/ npp.2009.104

34 Ramasubbu R, Lang S, Kiss ZHT. Dosing of electrical parameters in Deep Brain Stimulation (DBS) for intractable depression: a review of clinical studies. Front Psychiatry 2018;9:302. Doi: 10.3389/fpsyt.2018.00302

35 Lozano AM, Lipsman N. Probing and regulating dysfunctional circuits using deep brain stimulation. Neuron 2013;77(03): 406-424. Doi: 10.1016/j.neuron.2013.01.020

36 Tullberg M, Fletcher E, DeCarli C, et al. White matter lesions impair frontal lobe function regardless of their location. Neurology 2004; 63(02):246-253. Doi: 10.1212/01.WNL.0000130530.55104.B5

37 Hamani C, Mayberg H, Stone S, Laxton A, Haber S, Lozano AM. The subcallosal cingulate gyrus in the context of major depression. Biol Psychiatry 2011;69(04):301-308. Doi: 10.1016/j.biopsych. 2010.09.034

38 Haber SN, Kim KS, Mailly P, Calzavara R. Reward-related cortical inputs define a large striatal region in primates that interface with associative cortical connections, providing a substrate for incentive-based learning. J Neurosci 2006;26(32):8368-8376. Doi: 10.1523/JNEUROSCI.0271-06.2006

39 Mayberg HS, Brannan SK, Tekell JL, et al. Regional metabolic effects of fluoxetine in major depression: serial changes and relationship to clinical response. Biol Psychiatry 2000;48(08): 830-843. Doi: 10.1016/S0006-3223(00)01036-2

40 van den Munckhof P, Bosch DA, Mantione MHM, Figee M, Denys DAJP, Schuurman PR. Active stimulation site of nucleus accumbens deep brain stimulation in obsessive-compulsive disorder is localized in the ventral internal capsule. Acta Neurochir Suppl (Wien) 2013;117:53-59. Doi: 10.1007/978-3-7091-1482-7_9

41 Parent A, Carpenter MB. Carpenter's human neuroanatomy. 9th ed. Baltimore: Williams \& Wilkins; 1996 
42 Mogenson GJ, Swanson LW, Wu M. Neural projections from nucleus accumbens to globus pallidus, substantia innominata, and lateral preoptic-lateral hypothalamic area: an anatomical and electrophysiological investigation in the rat. J Neurosci 1983;3(01):189-202. Doi: 10.1523/JNEUROSCI.03-01-00189.1983

43 Schlaepfer TE, Cohen MX, Frick C, et al. Deep brain stimulation to reward circuitry alleviates anhedonia in refractory major depression. Neuropsychopharmacology 2008;33(02):368-377. Doi: 10.1038/sj.npp.1301408

44 Blomstedt P, Naesström M, Bodlund O. Deep brain stimulation in the bed nucleus of the stria terminalis and medial forebrain bundle in a patient with major depressive disorder and anorexia nervosa. Clin Case Rep 2017;5(05):679-684. Doi: 10.1002/ ccr3.856

45 Luyten L, Hendrickx S, Raymaekers S, Gabriëls L, Nuttin B. Electrical stimulation in the bed nucleus of the stria terminalis alleviates severe obsessive-compulsive disorder. Mol Psychiatry 2016;21(09):1272-1280. Doi: 10.1038/mp.2015.124

46 Wise RA. Forebrain substrates of reward and motivation. J Comp Neurol 2005;493(01):115-121. Doi: 10.1002/cne.20689

47 Zahm DS. The evolving theory of basal forebrain functionalanatomical 'macrosystems'. Neurosci Biobehav Rev 2006;30 (02):148-172. Doi: 10.1016/j.neubiorev.2005.06.003

48 Coenen VA, Panksepp J, Hurwitz TA, Urbach H, Mädler B. Human medial forebrain bundle (MFB) and anterior thalamic radiation (ATR): imaging of two major subcortical pathways and the dynamic balance of opposite affects in understanding depression. J Neuropsychiatry Clin Neurosci 2012;24(02):223-236. Doi: 10.1176/appi.neuropsych. 11080180

49 Dandekar MP, Fenoy AJ, Carvalho AF, Soares JC, Quevedo J. Deep brain stimulation for treatment-resistant depression: an integrative review of preclinical and clinical findings and translational implications. Mol Psychiatry 2018;23(05):1094-1112. Doi: $10.1038 / \mathrm{mp} .2018 .2$

50 Jiménez F, Nicolini H, Lozano AM, Piedimonte F, Salín R, Velasco F. Electrical stimulation of the inferior thalamic peduncle in the treatment of major depression and obsessive compulsive disorders. World Neurosurg 2013;80(3-4):30.e17-30.e25. DOI: 10.1016/j.wneu.2012.07.010

51 Winter C, Vollmayr B, Djodari-Irani A, Klein J, Sartorius A. Pharmacological inhibition of the lateral habenula improves depressive-like behavior in an animal model of treatment resistant depression. Behav Brain Res 2011;216(01):463-465. Doi: 10.1016/j.bbr.2010.07.034

52 Shumake J, Edwards E, Gonzalez-Lima F. Opposite metabolic changes in the habenula and ventral tegmental area of a genetic model of helpless behavior. Brain Res 2003;963(1-2):274-281. Doi: 10.1016/S0006-8993(02)04048-9

53 Schneider TM, Beynon C, Sartorius A, Unterberg AW, Kiening KL. Deep brain stimulation of the lateral habenular complex in treatment-resistant depression: traps and pitfalls of trajectory choice. Neurosurgery 2013;72(2, Suppl Operative)ons184-ons193, discussion ons193. Doi: 10.1227/NEU.0b013e318277a5aa

54 Yang Y, Wang H, Hu J, Hu H. Lateral habenula in the pathophysiology of depression. Curr Opin Neurobiol 2018;48:90-96. Doi: 10.1016/j.conb.2017.10.024

55 Lawson RP, Nord CL, Seymour B, et al. Disrupted habenula function in major depression. Mol Psychiatry 2017;22(02): 202-208. Doi: 10.1038/mp.2016.81

56 Sartorius A, Henn FA. Deep brain stimulation of the lateral habenula in treatment resistant major depression. Med Hypotheses 2007;69(06):1305-1308. Doi: 10.1016/j.mehy.2007.03.021

57 Lozano AM, Mayberg HS, Giacobbe P, Hamani C, Craddock RC, Kennedy SH. Subcallosal cingulate gyrus deep brain stimulation for treatment-resistant depression. Biol Psychiatry 2008;64(06): 461-467. Doi: 10.1016/j.biopsych.2008.05.034

58 McNeely HE, Mayberg HS, Lozano AM, Kennedy SH. Neuropsychological impact of $\mathrm{Cg} 25$ deep brain stimulation for treatment- resistant depression: preliminary results over 12 months. J Nerv Ment Dis 2008;196(05):405-410. Doi: 10.1097/nmd.0b013e31 81710927

59 McNab JA, Voets NL, Jenkinson N, et al. Reduced limbic connections may contraindicate subgenual cingulate deep brain stimulation for intractable depression. J Neurosurg 2009;111(04): 780-784. Doi: 10.3171/2009.2.jns081299

60 Puigdemont D, Portella MJ, Pérez-Egea R, et al. Depressive relapse after initial response to subcallosal cingulate gyrusdeep brain stimulation in a patient with a treatment-resistant depression: electroconvulsive therapy as a feasible strategy. Biol Psychiatry 2009;66(05):e11-e12. Doi: 10.1016/j.biopsych. 2009.03.018

61 Guinjoan SM, Mayberg HS, Costanzo EY, et al. Asymmetrical contribution of brain structures to treatment-resistant depression as illustrated by effects of right subgenual cingulum stimulation. J Neuropsychiatry Clin Neurosci 2010;22(03):265-277. Doi: $10.1176 /$ jnp.2010.22.3.265

62 Holtzheimer PE III, Mayberg HS. Deep brain stimulation for treatment-resistant depression. Am J Psychiatry 2010;167(12): 1437-1444. Doi: 10.1176/appi.ajp.2010.10010141

63 Kennedy SH, Giacobbe P, Rizvi SJ, et al. Deep brain stimulation for treatment-resistant depression: follow-up after 3 to 6 years. Am J Psychiatry 2011;168(05):502-510. Doi: 10.1176/appi. ajp.2010. 10081187

64 Holtzheimer PE, Kelley ME, Gross RE, et al. Subcallosal cingulate deep brain stimulation for treatment-resistant unipolar and bipolar depression. Arch Gen Psychiatry 2012;69(02):150-158. Doi: 10.1001/archgenpsychiatry.2011.1456

65 Broadway JM, Holtzheimer PE, Hilimire MR, et al. Frontal theta cordance predicts 6-month antidepressant response to subcallosal cingulate deep brain stimulation for treatment-resistant depression: a pilot study. Neuropsychopharmacology 2012;37 (07):1764-1772. Doi: 10.1038/npp.2012.23

66 Puigdemont D, Pérez-Egea R, Portella MJ, et al. Deep brain stimulation of the subcallosal cingulate gyrus: further evidence in treatment-resistant major depression. Int J Neuropsychopharmacol 2012;15(01):121-133. Doi: 10.1017/S1461145711001 088

67 Hamani C, Giacobbe P, Diwan M, et al. Monoamine oxidase inhibitors potentiate the effects of deep brain stimulation. Am J Psychiatry 2012;169(12):1320-1321. Doi: 10.1176/appi. ajp.2012.12060754

68 Lozano AM, Giacobbe P, Hamani C, et al. A multicenter pilot study of subcallosal cingulate area deep brain stimulation for treatment-resistant depression. J Neurosurg 2012;116(02):315-322. Doi: 10.3171/2011.10.JNS102122

69 Merkl A, Schneider GH, Schönecker T, et al. Antidepressant effects after short-term and chronic stimulation of the subgenual cingulate gyrus in treatment-resistant depression. Exp Neurol 2013;249:160-168. Doi: 10.1016/j.expneurol.2013.08.017

70 Ramasubbu R, Anderson S, Haffenden A, Chavda S, Kiss ZH. Doubleblind optimization of subcallosal cingulate deep brain stimulation for treatment-resistant depression: a pilot study. J Psychiatry Neurosci 2013;38(05):325-332. Doi: 10.1503/jpn.120160

71 Hilimire MR, Mayberg HS, Holtzheimer PE, et al. Effects of subcallosal cingulate deep brain stimulation on negative selfbias in patients with treatment-resistant depression. Brain Stimul 2015;8(02):185-191. Doi: 10.1016/j.brs.2014.11.010

72 Martín-Blanco A, Serra-Blasco M, Pérez-Egea R, et al. Immediate cerebral metabolic changes induced by discontinuation of deep brain stimulation of subcallosal cingulate gyrus in treatmentresistant depression. J Affect Disord 2015;173:159-162. Doi: 10.1016/j.jad.2014.10.035

73 Puigdemont D, Portella M, Pérez-Egea R, et al. A randomized double-blind crossover trial of deep brain stimulation of the subcallosal cingulate gyrus in patients with treatment-resistant depression: a pilot study of relapse prevention. J Psychiatry Neurosci 2015;40(04):224-231. Doi: 10.1503/jpn.130295 
74 Serra-Blasco M, de Vita S, Rodríguez MR, et al. Cognitive functioning after deep brain stimulation in subcallosal cingulate gyrus for treatment-resistant depression: an exploratory study. Psychiatry Res 2015;225(03):341-346. Doi: 10.1016/j.psychres. 2014.11.076

75 Ramasubbu R, Vecchiarelli HA, Hill MN, Kiss ZHT. Brain-derived neurotrophic factor and subcallosal deep brain stimulation for refractory depression. World J Biol Psychiatry 2015;16(02): 135-138. Doi: 10.3109/15622975.2014.952775

76 Accolla EA, Aust S, Merkl A, et al. Deep brain stimulation of the posterior gyrus rectus region for treatment resistant depression. J Affect Disord 2016;194:33-37. Doi: 10.1016/j.jad. 2016.01.022

77 Richieri R, Borius PY, Lagrange G, et al. Unmasking partial seizure after deep brain stimulation for treatment-resistant depression: a case report. Brain Stimul 2016;9(04):636-638. Doi: 10.1016/j. brs.2016.05.001

78 Holtzheimer PE, Husain MM, Lisanby SH, et al. Subcallosal cingulate deep brain stimulation for treatment-resistant depression: a multisite, randomised, sham-controlled trial. Lancet Psychiatry 2017;4(11):839-849. Doi: 10.1016/S2215-0366(17) 30371-1

79 Riva-Posse P, Choi KS, Holtzheimer PE, et al. A connectomic approach for subcallosal cingulate deep brain stimulation surgery: prospective targeting in treatment-resistant depression. Mol Psychiatry 2018;23(04):843-849. Doi: 10.1038/mp. 2017.59

80 Eitan R, Fontaine D, Benoît M, et al. One year double blind study of high vs low frequency subcallosal cingulate stimulation for depression. J Psychiatr Res 2018;96:124-134. Doi: 10.1016/j. jpsychires.2017.09.026

81 Merkl A, Aust S, Schneider GH, et al. Deep brain stimulation of the subcallosal cingulate gyrus in patients with treatmentresistant depression: A double-blinded randomized controlled study and long-term follow-up in eight patients. J Affect Disord 2018;227:521-529. Doi: 10.1016/j.jad.2017.11.024

82 Kuhn J, Lenartz D, Huff W, et al. Remission of alcohol dependency following deep brain stimulation of the nucleus accumbens: valuable therapeutic implications? J Neurol Neurosurg Psychiatry 2007;78(10):1152-1153. Doi: 10.1136/jnnp.2006.113092

83 Malone DA Jr, Dougherty DD, Rezai AR, et al. Deep brain stimulation of the ventral capsule/ventral striatum for treatment-resistant depression. Biol Psychiatry 2009;65(04): 267-275. Doi: 10.1016/j.biopsych.2008.08.029

84 Malone DA Jr. Use of deep brain stimulation in treatmentresistant depression. Cleve Clin J Med 2010;77(Suppl 3): S77-S80. Doi: 10.3949/ccjm.77.s3.14

85 Strong DR, Haber SN, Tyrka AR, Bernier JA, Rassmussen SA, Greenberg BD. Reversible increase in smoking after withdrawal of ventral capsule/ventral striatum deep brain stimulation in a depressed smoker. J Addict Med 2012;6(01):94-95. Doi: 10.1097/ADM.0b013e318240acf5

86 Millet B, Jaafari N, Polosan M, et al. Limbic versus cognitive target for deep brain stimulation in treatment-resistant depression: accumbens more promising than caudate. Eur Neuropsychopharmacol 2014;24(08):1229-1239. Doi: 10.1016/j.euroneuro. 2014.05.006

87 Giordana B, Benoît M, Darmon N, Yelnik J, Millet B, Fontaine D. Acute and reproducible mood improvement due to nucleus accumbens deep brain stimulation. Brain Stimul 2015;8(04): 842-843. Doi: 10.1016/j.brs.2015.05.004

88 Bergfeld IO, Mantione M, Hoogendoorn MLC, et al. Deep brain stimulation of the ventral anterior limb of the internal capsule for treatment-resistant depression: a randomized clinical trial. JAMA Psychiatry 2016;73(05):456-464. Doi: 10.1001/jamapsychiatry. 2016.0152

89 Bergfeld IO, Mantione M, Hoogendoorn MLC, et al. Impact of deep brain stimulation of the ventral anterior limb of the internal capsule on cognition in depression. Psychol Med 2017;47(09):1647-1658. Doi: 10.1017/S0033291717000113

90 Raymaekers S, Luyten L, Bervoets C, Gabriëls L, Nuttin B. Deep brain stimulation for treatment-resistant major depressive disorder: a comparison of two targets and long-term follow-up. Transl Psychiatry 2017;7(10):e1251. Doi: 10.1038/tp.2017.66

91 Schlaepfer TE, Bewernick BH, Kayser S, Mädler B, Coenen VA. Rapid effects of deep brain stimulation for treatment-resistant major depression. Biol Psychiatry 2013;73(12):1204-1212. Doi: 10.1016/j.biopsych.2013.01.034

92 Bewernick BH, Kayser S, Gippert SM, Switala C, Coenen VA, Schlaepfer TE. Deep brain stimulation to the medial forebrain bundle for depression- long-term outcomes and a novel data analysis strategy. Brain Stimul 2017;10(03):664-671. Doi: 10.1016/j.brs.2017.01.581

93 Jiménez F, Velasco F, Salin-Pascual R, et al. A patient with a resistant major depression disorder treated with deep brain stimulation in the inferior thalamic peduncle. Neurosurgery 2005;57(03):585-593, discussion 585-593. Doi: 10.1227/01. NEU.0000170434.44335.19

94 Aouizerate B, Cuny E, Martin-Guehl C, et al. Deep brain stimulation of the ventral caudate nucleus in the treatment of obsessive-compulsive disorder and major depression. Case report. J Neurosurg 2004;101(04):682-686. Doi: 10.3171/ jns.2004.101.4.0682

95 Kosel M, Sturm V, Frick C, et al. Mood improvement after deep brain stimulation of the internal globus pallidus for tardive dyskinesia in a patient suffering from major depression. J Psychiatr Res 2007;41(09):801-803. Doi: 10.1016/j.jpsychires. 2006.07.010

96 Fitzgerald PB, Segrave R, Richardson KE, et al. A pilot study of bed nucleus of the stria terminalis deep brain stimulation in treatment-resistant depression. Brain Stimul 2018;11(04):921-928. Doi: 10.1016/j.brs.2018.04.013

97 Coenen VA, Sajonz B, Reisert M, et al. Tractography-assisted deep brain stimulation of the superolateral branch of the medial forebrain bundle (sIMFB DBS) in major depression. Neuroimage Clin 2018;20:580-593. Doi: 10.1016/j.nicl.2018.08.020

98 Bewernick BH, Hurlemann R, Matusch A, et al. Nucleus accumbens deep brain stimulation decreases ratings of depression and anxiety in treatment-resistant depression. Biol Psychiatry 2010; 67(02):110-116. Doi: 10.1016/j.biopsych.2009.09.013

99 Grubert C, Hurlemann R, Bewernick BH, et al. Neuropsychological safety of nucleus accumbens deep brain stimulation for major depression: effects of 12-month stimulation. World J Biol Psychiatry 2011;12(07):516-527. Doi: 10.3109/15622975.2011.583940

100 Bewernick BH, Kayser S, Sturm V, Schlaepfer TE. Long-term effects of nucleus accumbens deep brain stimulation in treatment-resistant depression: evidence for sustained efficacy. Neuropsychopharmacology 2012;37(09):1975-1985. Doi: 10.1038/npp.2012.44

101 Fenoy AJ, Schulz PE, Selvaraj S, et al. A longitudinal study on deep brain stimulation of the medial forebrain bundle for treatmentresistant depression. Transl Psychiatry 2018;8(01):111. Doi: 10.1038/s41398-018-0160-4

102 Fins JJ, Kubu CS, Mayberg HS, Merkel R, Nuttin B, Schlaepfer TE. Being open minded about neuromodulation trials: Finding success in our "failures". Brain Stimul 2017;10(02):181-186. Doi: 10.1016/j.brs.2016.12.012

103 Park SC, Kim JM, Jun TY, et al. How many different symptom combinations fulfil the diagnostic criteria for major depressive disorder? Results from the CRESCEND study. Nord J Psychiatry 2017;71(03):217-222. Doi: 10.1080/08039488.2016.1265584

104 Thase ME, Rush AJ. When at first you don't succeed: sequential strategies for antidepressant nonresponders. J Clin Psychiatry 1997;58(Suppl 13):23-29

105 Zhang C, Zhang Y, Zhan S, et al. Telemedical deep brain stimulation: merits and limitations. Stereotact Funct Neurosurg 2018; 96(04):272-273. Doi: 10.1159/000491603 
106 Naudet F, Millet B, Michel Reymann J, Falissard B. The fallacy of thresholds used in defining response and remission in depression rating scales. Int J Methods Psychiatr Res 2014;23(04): 469-473. Doi: $10.1002 / \mathrm{mpr} .1393$

107 Kadouri A, Corruble E, Falissard B. The improved Clinical Global Impression Scale (iCGI): development and validation in depression. BMC Psychiatry 2007;7:7. Doi: 10.1186/1471244X-7-7

108 Gardner J. A history of deep brain stimulation: technological innovation and the role of clinical assessment tools. Soc Stud Sci 2013;43(05):707-728. Doi: 10.1177/0306312713483678

109 Coenen VA, Schlaepfer TE, Maedler B, Panksepp J. Cross-species affective functions of the medial forebrain bundle-implications for the treatment of affective pain and depression in humans. Neurosci Biobehav Rev 2011;35(09):1971-1981. Doi: 10.1016/j. neubiorev.2010.12.009

110 Mestre TA, Lang AE, Okun MS. Factors influencing the outcome of deep brain stimulation: Placebo, nocebo, lessebo, and lesion effects. Mov Disord 2016;31(03):290-296. Doi: 10.1002/mds.26500

111 Wulsin LR, Vaillant GE, Wells VE. A systematic review of the mortality of depression. Psychosom Med 1999;61(01):6-17
112 Narasimhan M, Raynor JD, Jones AB. Depression in the medically ill: diagnostic and therapeutic implications. Curr Psychiatry Rep 2008;10(03):272-279. Doi: 10.1007/s11920-008-0044-z

113 Priori A, Foffani G, Rossi L, Marceglia S. Adaptive deep brain stimulation (aDBS) controlled by local field potential oscillations. Exp Neurol 2013;245:77-86. Doi: 10.1016/j.expneurol.2012.09.013

114 Nuttin B, Wu H, Mayberg H, et al. Consensus on guidelines for stereotactic neurosurgery for psychiatric disorders. J Neurol Neurosurg Psychiatry 2014;85(09):1003-1008. Doi: 10.1136/ jnnp-2013-306580

115 Case A, Deaton A. Rising morbidity and mortality in midlife among white non-Hispanic Americans in the 21st century. Proc Natl Acad Sci U S A 2015;112(49):15078-15083. Doi: 10.1073/ pnas. 1518393112

116 Bewernick B, Schlaepfer TE. Update on neuromodulation for treatment-resistant depression. F1000 Res 2015;4:1000. Doi: 10.12688/f1000research.6633.1

117 Stroupe KT, Weaver FM, Cao L, et al. Cost of deep brain stimulation for the treatment of Parkinson's disease by surgical stimulation sites. Mov Disord 2014;29(13):1666-1674. Doi: 10.1002/ mds.26029 